Алгебра и анализ

Том 21 (2009), № 5
St. Petersburg Math. J.

Vol. 21 (2010), No. 5, Pages 791-818 S 1061-0022(2010)01118-X

Article electronically published on July 15, 2010

\title{
THE ESHELBY THEOREM AND PATCH OPTIMIZATION PROBLEM
}

\author{
S. A. NAZAROV
}

\begin{abstract}
Let $\Omega^{0}$ be an ellipsoidal inclusion in the Euclidean space $\mathbb{R}^{n}$. It is checked that if a solution of the homogeneous transmission problem for a formally selfadjoint elliptic system of second order differential equations with piecewise smooth coefficients grows linearly at infinity, then this solution is a linear vector-valued function in the interior of $\Omega^{0}$. This fact generalizes the classical Eshelby theorem in elasticity theory and makes it possible to indicate simple and explicit formulas for the polarization matrix of the inclusion in the composite space, as well as to solve a problem about optimal patching of an elliptical hole.
\end{abstract}

\section{$\S 1$. Setting of the transmission PROBLEM}

Let $\Omega^{0}$ be a nonempty domain in the Euclidean space $\mathbb{R}^{n}, n \geq 2$, with a smooth (of class $C^{\infty}$ ) boundary, and let $\Omega^{1}=\mathbb{R}^{n} \backslash \overline{\Omega^{0}}$ be its exterior. Suppose it has compact closure: $\overline{\Omega^{0}}=\partial \Omega^{0} \cup \Omega^{0} \subset \mathbb{B}_{R}=\{x:|x|<R\}, R>0$. We place the origin of a Cartesian coordinate system $x=\left(x_{1}, \ldots, x_{n}\right)$ in the interior of $\Omega^{0}$. Let $\mathcal{D}\left(\nabla_{x}\right)$ be an $(N \times k)$-matrix of first order differential operators with constant and, in general, complex coefficients, and let $\mathcal{A}^{i}, i=0,1$, be numerical Hermitian positive definite matrices of size $N \times N$. We introduce the formally selfadjoint $(k \times k)$-matrices of second order differential operators

$$
\mathcal{L}^{i}\left(\nabla_{x}\right)={\overline{\mathcal{D}\left(-\nabla_{x}\right)}}^{\top} \mathcal{A}^{i} \mathcal{D}\left(\nabla_{x}\right)
$$

and consider the following system of differential equations with transmission conditions on the interface $\Gamma=\partial \Omega^{0}=\partial \Omega^{1}$ :

$$
\begin{aligned}
& \mathcal{L}^{i}\left(\nabla_{x}\right) u^{i}(x)=f^{i}(x), \quad x \in \Omega^{i}, i=0,1, \\
& u^{0}(x)-u^{1}(x)=0, \quad \mathcal{N}^{0}\left(x, \nabla_{x}\right) u^{0}(x)-\mathcal{N}^{1}\left(x, \nabla_{x}\right) u^{1}(x)=g(x), \quad x \in \Gamma .
\end{aligned}
$$

Our problem needs a more detailed description.

To start with, we clarify the notation. By $u^{i}$ we mean the restrictions of the vectorvalued function $u=\left(u_{1}, \ldots, u_{k}\right)^{\top}$ to the sets $\Omega^{i}$; this function, as well as the righthand sides $f, g$ of the problem, are viewed as columns, i.e., $\top$ means transposition. Also, $\nabla_{x}=\operatorname{grad}$ and the bar means complex conjugation, so that ${\overline{\mathcal{D}\left(-\nabla_{x}\right)}}^{\top}$ is the matrix differential operator formally adjoint to $\mathcal{D}\left(\nabla_{x}\right)$. Finally, $\mathcal{N}^{i}$ is the operator of the Neumann boundary conditions,

$$
\mathcal{N}^{i}\left(x, \nabla_{x}\right)=\overline{\mathcal{D}(\nu(x))}^{\top} \mathcal{A}^{i} \mathcal{D}\left(\nabla_{x}\right),
$$

and $\nu(x)$ is the outward unit normal (column) on the boundary $\Gamma$ of $\Omega^{0}$.

2010 Mathematics Subject Classification. Primary 35J57, 74B05.

Key words and phrases. Formally selfadjoint elliptic system, junction conditions, ellipsoidal inclusion, Eshelby theorem, optimization of inclusion.

Supported by RFBR (grant no. 09-01-00759). 
All the subsequent considerations equally pertain also to the outer Neumann problem

$$
\begin{aligned}
\mathcal{L}^{1}\left(\nabla_{x}\right) u^{1}(x) & =f^{1}(x), \quad x \in \Omega^{1}, \\
-\mathcal{N}^{1}\left(x, \nabla_{x}\right) u^{1}(x) & =g(x), \quad x \in \Gamma,
\end{aligned}
$$

which can be obtained from problem (1.2), (1.3) by limit passage as $\mathcal{A}^{0} \rightarrow 0 \in \mathbb{C}^{N \times N}$. We emphasize that the minus sign on the left-hand side of the boundary condition is caused by the fact that $\nu$ is the inward normal for the domain $\Omega^{1}$. In Remark 4.4, we shall discuss the limit passage as $\mathcal{A}^{0} \rightarrow \infty \in \mathbb{C}^{N \times N}$.

Now we list the requirements imposed on the matrix $\mathcal{D}\left(\nabla_{x}\right)$. It is assumed to be algebraically complete [1]: there is a number $\varrho_{\mathcal{D}} \in \mathbb{N}:=\{1,2, \ldots\}$ such that for any row $p(\xi)=\left(p_{1}(\xi), \ldots, p_{k}(\xi)\right)$ of homogeneous polynomials of degree $\varrho \geq \varrho_{\mathcal{D}}$ in the variables $\xi=\left(\xi_{1}, \ldots, \xi_{n}\right)^{\top}$ there exists a polynomial row $q(\xi)=\left(q_{1}(\xi), \ldots, q_{N}(\xi)\right)$ for which

$$
p(\xi)=q(\xi) \mathcal{D}(\xi), \quad \xi \in \mathbb{R}^{n} .
$$

In this case, the formally selfadjoint operator (1.1) is said to be formally positive and turns out to be elliptic, and the pair $\left\{\mathcal{L}^{i}, \mathcal{N}^{i}\right\}$ satisfies the Shapiro-Lopatinsky conditions (see [1] and 2, 3]). Also, the corresponding Hermitian sesquilinear forms

$$
\begin{aligned}
a^{i}\left(u^{i}, v^{i} ; \Omega^{i}\right) & =\left(\mathcal{A}^{i} \mathcal{D}\left(\nabla_{x}\right) u^{i}, \mathcal{D}\left(\nabla_{x}\right) v^{i}\right)_{\Omega^{i}}, \quad i=0,1, \\
a\left(u, v ; \mathbb{R}^{n}\right) & =a^{0}\left(u^{0}, v^{0} ; \Omega^{0}\right)+a^{1}\left(u^{1}, v^{1} ; \Omega^{1}\right)
\end{aligned}
$$

possess the polynomial property [2, 3]:

$$
a(u, u ; \Xi)=0,\left.u \in C^{1}(\bar{\Xi})^{k} \quad \Leftrightarrow \quad u \in \mathcal{P}\right|_{\Xi} .
$$

Here $\Xi$ is an arbitrary domain in $\mathbb{R}^{n}$ and $\mathcal{P}$ is a finite-dimensional linear space of vectorvalued polynomials. With the help of (1.6), it is not hard to verify (see, e.g., 2, 3]) that the degrees of the scalar polynomials $p_{j}$ in the linear space

$$
\mathcal{P}=\left\{p=\left(p_{1}, \ldots, p_{k}\right)^{\top}: \mathcal{D}\left(\nabla_{x}\right) p=0\right\}
$$

do not exceed $\varrho_{\mathcal{D}}-1$. By $(,)_{\Omega}$ we mean the natural scalar product in the Lebesgue space $L_{2}(\Omega)^{m}$; the index $m$ indicates the number of components of vector-valued functions, but we do not write it in the notation for scalar products and norms.

In the survey 3 , the reader can find many examples of specific problems of mathematical physics that possess the properties required above. Here we restrict ourselves to two of them, discussed below.

Example 1.1. Suppose $k=1, N=n$, and $\mathcal{D}\left(\nabla_{x}\right)=\nabla_{x}$. Then $\mathcal{L}^{i}\left(\nabla_{x}\right)=-\nabla_{x}^{\top} \mathcal{A}^{i} \nabla_{x}$ is a scalar operator in divergence form, and $\varrho_{\mathcal{D}}=1, \mathcal{P}=\mathbb{C}$.

Example 1.2. For the 3-dimensional $(n=3)$ linearized system of elasticity theory that describes the deformation of an anisotropic and nonhomogeneous composite body, the operators (1.1), written in the matrix (rather than tensor; see, e.g., [4, 5]) form, are composed of real $(6 \times 6)$-matrices of elastic modules and a $(6 \times 3)$-matrix $\mathcal{D}\left(\nabla_{x}\right)$,

$$
\mathcal{D}(\xi)=\left(\begin{array}{cccccc}
\xi_{1} & 0 & 0 & 0 & 2^{-1 / 2} \xi_{3} & 2^{1 / 2} \xi_{2} \\
0 & \xi_{2} & 0 & 2^{-1 / 2} \xi_{3} & 0 & 2^{-1 / 2} \xi_{1} \\
0 & 0 & \xi_{3} & 2^{-1 / 2} \xi_{2} & 2^{-1 / 2} \xi_{1} & 0
\end{array}\right)^{\top} .
$$

It is not hard to check that the matrix (1.6) is algebraically complete (see (1.10) ); in particular, $k=3, N=6$, and $\varrho_{\mathcal{D}}=2$. As to the polynomial property (1.8), it involves the linear space

$$
\mathcal{P}=\left\{p(x)=d(x) a: a=\left(a_{1}, a_{2}, a_{3}, a_{4}, a_{5}, a_{6}\right)^{\top} \in \mathbb{R}^{6}\right\},
$$


spanned by the columns of the $(3 \times 6)$-matrix

$$
d(x)=\left(\begin{array}{cccccc}
1 & 0 & 0 & 0 & 2^{-1 / 2} x_{3} & -2^{1 / 2} x_{2} \\
0 & 1 & 0 & -2^{-1 / 2} x_{3} & 0 & 2^{-1 / 2} x_{1} \\
0 & 0 & 1 & 2^{-1 / 2} x_{2} & -2^{-1 / 2} x_{1} & 0
\end{array}\right) .
$$

The vectors $d(x) a$ are rigid motions that are translational $\left(a_{4}=a_{5}=a_{6}=0\right)$ or rotational $\left(a_{1}=a_{2}=a_{3}=0\right)$. Due to the factors $2^{-1 / 2}$, the column of deformations

$$
\varepsilon(u):=\mathcal{D}\left(\nabla_{x}\right) u=\left(\varepsilon_{11}(u), \varepsilon_{22}(u), \varepsilon_{33}(u), 2^{1 / 2} \varepsilon_{23}(u), 2^{1 / 2} \varepsilon_{31}(u), 2^{1 / 2} \varepsilon_{12}(u)\right)^{\top}
$$

(see [5, Chapter 2]) has the same natural norm as the strain tensor $\left(\varepsilon_{j k}(u)\right)_{j, k=1}^{3}$ with the components $\varepsilon_{j k}(u)=\frac{1}{2}\left(\frac{\partial u_{j}}{\partial x_{k}}+\frac{\partial u_{k}}{\partial x_{j}}\right)$. The column $\sigma^{i}(u):=\mathcal{A}^{i} \mathcal{D}\left(\nabla_{x}\right) u^{i}$ has a structure similar to (1.12) and contains components of the stress tensor. The matrix $\mathcal{A}^{i}$, which relates the columns of stresses and strains, is called the stiffness matrix; its symmetry and positive definiteness are prescribed by the nature of the elastic medium.

Example 1.3. In the 2-dimensional $(n=2)$ problem of elasticity theory, the operators (1.1) of size $2 \times 2$ are built out of the $(3 \times 3)$-matrices $\mathcal{A}^{i}$ and $(3 \times 2)$-matrix $\mathcal{D}\left(\nabla_{x}\right)$,

$$
\mathcal{D}(\xi)=\left(\begin{array}{ccc}
\xi_{1} & 0 & 2^{1 / 2} \xi_{2} \\
0 & \xi_{2} & 2^{-1 / 2} \xi_{1}
\end{array}\right)^{\top}
$$

In this setting, $\operatorname{dim} \mathcal{P}=3$,

$$
\mathcal{P}=\left\{p(x)=d(x) a: a=\left(a_{1}, a_{2}, a_{3}\right)^{\top} \in \mathbb{R}^{3}\right\}, \quad d(x)=\left(\begin{array}{ccc}
1 & 0 & -2^{1 / 2} x_{2} \\
0 & 1 & 2^{-1 / 2} x_{1}
\end{array}\right),
$$

and

$$
\begin{aligned}
\varepsilon(u) & =\mathcal{D}\left(\nabla_{x}\right) u=\left(\varepsilon_{11}(u), \varepsilon_{22}(u), 2^{1 / 2} \varepsilon_{12}(u)\right)^{\top}, \\
\sigma^{i}(u) & =\mathcal{A}^{i} \mathcal{D}\left(\nabla_{x}\right) u^{i}=\left(\sigma_{11}^{i}(u), \sigma_{22}^{i}(u), 2^{1 / 2} \sigma_{12}^{i}(u)\right)^{\top}
\end{aligned}
$$

are columns of strains and stresses, respectively. For an isotropic medium, the stiffness matrices have the form

$$
\mathcal{A}^{i}=\left(\begin{array}{ccc}
\boldsymbol{\lambda}^{i}+2 \boldsymbol{\mu}^{i} & \boldsymbol{\lambda}^{i} & 0 \\
\boldsymbol{\lambda}^{i} & \boldsymbol{\lambda}^{i}+2 \boldsymbol{\mu}^{i} & 0 \\
0 & 0 & 2 \boldsymbol{\mu}^{i}
\end{array}\right)
$$

where $\boldsymbol{\lambda}^{i} \geq 0$ and $\boldsymbol{\mu}^{i}>0$ are the Lamé constants.

\section{§2. Preliminary description of Results}

In $\S 3$, we study the solvability of problem (1.2), (1.3) and the asymptotics of its solutions. After that, in $\S 4$, we define an integral characteristic of the inclusion $\Omega^{0}$ in the composite space $\Omega^{1}$, namely, the polarization matrix $P$ (see [6] and [7, 8] for scalar problems and elasticity theory problems, respectively, and also [9] for general formally selfadjoint elliptic systems). This characteristic occurs in asymptotic formulas for various functionals calculated at the solution of the singularly perturbed boundaryvalue problems

$$
\begin{gathered}
\mathcal{L}^{1}\left(\nabla_{x}\right) v_{(h)}^{1}(x)=f(x), \quad x \in \Xi(h), \quad \mathcal{L}^{0}\left(\nabla_{x}\right) v_{(h)}^{0}(x)=f(x), \quad x \in \omega_{h}, \\
v_{(h)}^{0}(x)-v_{(h)}^{1}(x)=0, \quad \mathcal{N}^{0}\left(x, \nabla_{x}\right) v_{(h)}^{0}(x)-\mathcal{N}^{1}\left(x, \nabla_{x}\right) v_{(h)}^{1}(x)=0, \quad x \in \partial \omega_{h}, \\
v_{(h)}^{0}(x)=0, \quad x \in \partial \Xi,
\end{gathered}
$$

in the composite domain $\Xi(h) \cup \omega_{h}$; here $h \in\left(0, h_{0}\right]$ is a small parameter, $h_{0}>0$,

$$
\omega_{h}=\left\{x \in \mathbb{R}^{n}: \xi:=h^{-1} x \in \Omega^{0}\right\} \subset \Xi \text { for } h \in\left(0, h_{0}\right], \quad \Xi(h)=\Xi \backslash \overline{\omega_{h}},
$$




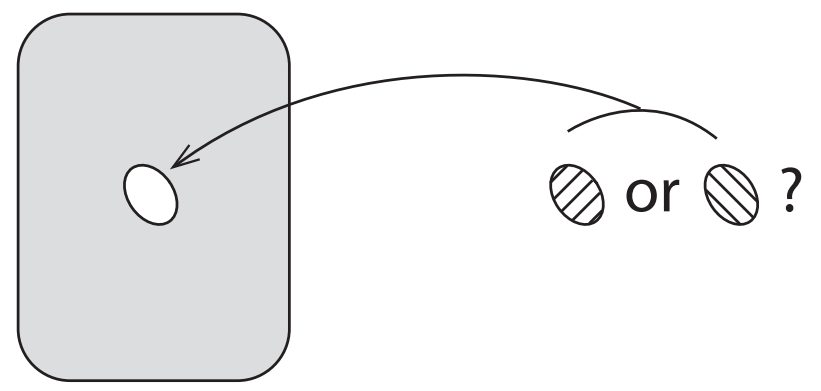

Figure 1. Patch optimization.

and $\Xi$ is a domain in $\mathbb{R}^{n}$ with smooth boundary $\partial \Xi$ and compact closure $\bar{\Xi}=\Xi \cup \partial \Xi$; the origin of the $x$ coordinates is assumed to lie inside $\Xi$. Also, we assume that the origin of the $\xi$ coordinates is in the interior of $\omega$.

The polarization matrix admits two descriptions: it is formed by the coefficients in the expansions at infinity of special solutions of problem (1.2), (1.3) and is the Gram matrix constructed by these solutions with the help of the scalar product

$$
\langle u, v\rangle=\left(\mathcal{A D}\left(\nabla_{x}\right) u, \mathcal{D}\left(\nabla_{x}\right) v\right)_{\mathbb{R}^{n}}
$$

(see the proof of Proposition 3.1).

Explicit formulas for the polarization matrix are known for some specific scalar differential equations and systems of mathematical physics in the case of the canonical cavitie 1 , and therefore, the optimization problem for the cavity's shape or for the shape and the physical properties of an inclusion becomes fairly difficult in general. In elasticity theory, to study the properties of composite bodies (see Examples 1.2 and 1.3), the classical Eshelby theorem is used, which states that inside the ellipsoidal inclusion

$$
\Omega=\left\{x \in \mathbb{R}^{n}: \frac{x_{1}^{2}}{\ell_{1}^{2}}+\cdots+\frac{x_{n}^{2}}{\ell_{n}^{2}}=1\right\}, \quad \ell_{j}>0,
$$

the deformation field $\varepsilon^{0}=\mathcal{D}\left(\nabla_{x}\right) u^{0}$ generated by the constant stresses $\sigma^{\infty}$ (or the strains $\left.\varepsilon^{\infty}=\left(\mathcal{A}^{1}\right)^{-1} \sigma^{\infty}\right)$ at infinity turns out to be constant. This theorem, proved for isotropic media in the original paper [14, was discussed, applied, and generalized to anisotropic media and polynomial loadings in a huge number of publications (see the books [15, 16, the papers [17, 18, 19, 20, 21], and the references therein). Nevertheless, in the literature, the author could not find any purely mathematical justification of the claim stated, without gaps admissible only at a physical level of rigor: inversion of matrices without verification that they are nonsingular, regularization of a singular integral operator after compilation of the corresponding equation, and so on. Therefore, one of our main goals in the present paper became the proof of Theorem [5.1 (see $\S 5$ ) for the transmission problem (1.2), (1.3) in the case of the ellipsoid (2.6), and also the introduction of canonical objects such as the link matrix $T$ in the formula

$$
\varepsilon^{0}=\left(\mathbb{I}_{N}+T\left(\mathcal{A}^{0}-\mathcal{A}^{1}\right)\right) \varepsilon^{\infty} .
$$

It should be emphasized that $T$ is a Hermitian positive definite matrix for any $\mathcal{A}^{0}$ and $\mathcal{A}^{1}$. As a by-product, the relationship (5.23) was found between the polarization matrix $P$ and the link matrix $T$, which allowed us in $\S 6$ to construct a simple algorithm that solves an optimal patch problem: having a small ellipsoidal hole $\omega_{h}$ (see formulas (2.4),

\footnotetext{
${ }^{1}$ The simple formulas, suggested in 10 and replicated in [11, 12, for the polarization matrix in the plane problem of elasticity theory are wrong: a mistake in [10] was found in [13], but its amendment made the final formulas bulky and completely implicit.
} 
(2.6) and Figure 1) in a body $G$ with the matrix $\mathcal{A}^{1}$, it is required to cut a patch $\omega_{h}$ out of a material with the matrix $\mathcal{A}^{0}$ so that the energy functional

$$
E\left(v_{(h)} ; f\right)=\frac{1}{2} a\left(v_{(h)}, v_{(h)} ; \Xi\right)-\left(f, v_{(h)}\right)_{\Xi}=-\frac{1}{2}\left(f, v_{(h)}\right)_{\Xi}
$$

take its minimal (maximal) value. In other words, the shape of the patch is fixed, and we must relate the position of the ellipsoid $\omega_{h}$ to the axes of anisotropy of the patch's material, which is assumed to be homogeneous, but anisotropic (the coefficient matrix $\mathcal{A}^{0}$ is constant, but arbitrary). In formula (2.8), $v_{(h)}$ is a weak solution of problem (2.1) - (2.3) belonging to the Sobolev space $\stackrel{H}{H}^{1}(\Xi)^{k}$ (the symbol ${ }^{\circ}$ points to the Dirichlet condition (2.3)). In Theorem 6.1 we give an explicit solution of the scalar version of the patch problem.

\section{§3. Solvability of THE TRAnSmission PROBlem}

Due to the structure of the differential operators (1.1), (1.4) and the sesquilinear forms (1.7), the Green formula is valid,

$$
\left(\mathcal{L}^{0} u^{0}, v^{0}\right)_{\Omega^{0}}+\left(\mathcal{L}^{1} u^{1}, v^{1}\right)_{\Omega^{1}}+\left(\mathcal{N}^{0} u^{0}, v^{0}\right)_{\Gamma}-\left(\mathcal{N}^{1} u^{1}, v^{1}\right)_{\Gamma}=a\left(u, v ; \mathbb{R}^{n}\right),
$$

where $u, v \in C_{c}^{0}\left(\mathbb{R}^{n}\right)^{k} \cap C^{1}\left(\overline{\Omega^{0}} \cup \overline{\Omega^{1}}\right)^{k}$ are compactly supported vector-valued functions continuous on $\mathbb{R}^{n}$ and continuously differentiable in the domains $\Omega^{i}$ up to the boundary $\Gamma$. The minus sign of the last scalar product on the left in (3.1) is explained by the same reason as in the boundary conditions (1.5). Thus, the variational setting [22, 23 ] of problem (1.2), (1.3) looks as follows:

$$
a\left(u, v ; \mathbb{R}^{n}\right)=(f, v)_{\mathbb{R}^{n}}+(g, v)_{\Gamma}, \quad v \in \mathcal{H} .
$$

The natural energy space $\mathcal{H}$ is the closure of the linear space $C_{c}^{1}\left(\mathbb{R}^{n}\right)^{k}$, e.g., in the norm $\left(\left\|\nabla_{x} u ; L_{2}\left(\mathbb{R}^{n}\right)\right\|^{2}+\left\|u ; L_{2}\left(\Omega^{0}\right)\right\|^{2}\right)^{1 / 2}$. In the proposition below it will be shown that the norm in $\mathcal{H}$ can be given by the formula

$$
\|u ; \mathcal{H}\|=\left(\left\|\nabla_{x} u ; L_{2}\left(\mathbb{R}^{n}\right)\right\|^{2}+\left\|\rho^{-1} u ; L_{2}\left(\mathbb{R}^{n}\right)\right\|^{2}\right)^{1 / 2},
$$

with the weight factor

$$
\rho(x)= \begin{cases}1+|x| & \text { if } n>2 \\ (1+|x|)(1+|\ln | x||) & \text { if } n=2\end{cases}
$$

Proposition 3.1. Suppose that

$$
\rho f \in L_{2}\left(\mathbb{R}^{n}\right)^{k}, \quad g \in L_{2}(\Gamma)^{k},
$$

Also, if $n=2$, we assume the orthogonality conditions

$$
\int_{\mathbb{R}^{n}} f(x) d x+\int_{\Gamma} g(x) d s_{x}=0 \in \mathbb{C}^{k} .
$$

Then the variational problem (3.2) has a solutions $u \in \mathcal{H}$ (a weak solution of problem (1.2), (1.3) ), which turns out to be unique if $n>2$ and is determined up to a constant summand in $\mathbb{C}^{k}$ if $n=2$.

Proof. The algebraic completeness of the matrix $\mathcal{D}$ implies the inequality

$$
\left\|\nabla_{x} v ; L_{2}\left(\mathbb{R}^{n}\right)\right\| \leq c_{\mathcal{D}}\left\|\mathcal{D}\left(\nabla_{x}\right) v ; L_{2}\left(\mathbb{R}^{n}\right)\right\|, \quad v \in C_{c}^{1}\left(\mathbb{R}^{n}\right)^{k},
$$

with some constant $c_{\mathcal{D}}$. Such an inequality was established in [1, Theorem 7.3.6, p. 191] even for an arbitrary domain bounded by a Lipschitzian surface; however, since in the case of an unbounded domain the proof is simple, we present it here for the reader's convenience. 
Applying the Fourier transformation $v(x) \mapsto \widehat{v}(\xi)$ and the Parseval identity, we obtain

We check that

$$
\left\|\mathcal{D}\left(\nabla_{x}\right) v ; L_{2}(\Omega)\right\|^{2}=C \int_{\mathbb{R}^{n}}|\mathcal{D}(\xi) \widehat{v}(\xi)|^{2} d \xi
$$

$$
|\mathcal{D}(\xi) a|^{2} \geq c_{\mathcal{D}}|\xi|^{2}|a|^{2}, \quad a \in \mathbb{C}^{k}, \quad \xi \in \mathbb{R}^{n}, \quad c_{\mathcal{D}}>0
$$

then formula (3.8) implies (3.7) after the inverse Fourier transformation. Suppose inequality (3.9) fails, so that there exist nonzero columns $a^{0} \in \mathbb{C}^{k}$ and $\xi^{0} \in \mathbb{R}^{n}$ such that $\mathcal{D}\left(\xi^{0}\right) a^{0}=0$. Putting $p(\xi)=\left(a^{0}\right)^{\top}|\xi|^{2 \varrho_{\mathcal{D}}}$ in (1.6), we take the scalar product of this identity and the column $a^{0}$. For $\xi=\xi^{0}$ we have $\left|a^{0}\right|^{2}\left|\xi^{0}\right|^{2 \varrho \mathcal{D}}=q\left(\xi^{0}\right) \mathcal{D}\left(\xi^{0}\right) a^{0}=0$, which implies that either $a^{0}=0$ or $\xi^{0}=0$, a contradiction.

If $n \geq 3$, after passage to the spherical coordinates $(r, \varphi) \in(0, \infty) \times \mathbb{S}^{n-1}$ and integration over the unit sphere $\mathbb{S}^{n-1}$, the one-dimensional Hardy inequality

$$
\int_{0}^{\infty} r^{n-3}|U(r)|^{2} d r \leq \frac{4}{(n-2)^{2}} \int_{0}^{\infty} r^{n-1}\left|\frac{d U}{d r}(r)\right|^{2} d r, \quad U \in C_{c}^{1}[0, \infty),
$$

provides the estimate

$$
\left\||x|^{-1} u ; L_{2}\left(\mathbb{R}^{n}\right)\right\|^{2} \leq \frac{4}{(n-2)^{2}}\left\|\nabla_{x} u ; L_{2}\left(\mathbb{R}^{n}\right)\right\|^{2}, \quad u \in C_{c}^{1}\left(\mathbb{R}^{n}\right)^{k} .
$$

The weight factor $|x|^{-1}$ on the left is not less than $\rho(x)=(1+|x|)^{-1}$.

If $n=2$, then relations (3.10) and (3.11) are replaced with

$$
\begin{gathered}
\int_{\mathbf{r}}^{\infty} r^{-1}\left|\ln \frac{r}{\mathbf{r}}\right|^{-2}|U(r)|^{2} d r \leq 4 \int_{\mathbf{r}}^{\infty} r\left|\frac{d U}{d r}(r)\right|^{2} d r, \quad U \in C_{c}^{1}(\mathbf{r}, \infty), \quad \mathbf{r}>0, \\
\left\|\rho^{-1} u ; L_{2}\left(\mathbb{R}^{n}\right)\right\|^{2} \leq c\left(\left\|\nabla_{x} u ; L_{2}\left(\mathbb{R}^{n}\right)\right\|^{2}+\left\|u ; L_{2}\left(\Omega^{0}\right)\right\|^{2}\right), \quad u \in C_{c}^{1}\left(\mathbb{R}^{n}\right)^{k} .
\end{gathered}
$$

It should be emphasized that, before applying the "logarithmic" Hardy inequality (3.12), we should multiply $u$ by a cutoff function that vanishes for $|x| \leq \mathbf{r}$ (the norm $\left\|u ; L_{2}\left(\Omega^{0}\right)\right\|$ on the right in (3.13) emerges precisely for this reason), and the radius $\mathbf{r}$ is chosen so as to ensure that $\mathbb{B}_{\mathbf{r}} \subset \Omega^{0}$. This leads to important consequences: unlike the case where $n \geq 3$, for $n=2$ the expression (2.5) is a scalar product in the quotient space $\mathcal{H} / \mathbb{C}^{k}$ rather than in the energy space $\mathcal{H}$ with the norm (3.3). In order to check that the constant columns fall within the closure $\mathcal{H}$ of $C_{c}^{1}(\mathbb{R})^{k}$ in the norm (3.3), consider the sequence of vector-valued functions $b \chi\left(|\ln m|^{-1} \ln |x|\right)$, where $m \in \mathbb{N}, b \in \mathbb{C}^{k}$, and $\chi \in C^{\infty}(\mathbb{R})$ is a standard cutoff function,

$$
\chi(t)=0 \text { for } t \geq 1, \quad \chi(t)=1 \text { for } t \leq 1 / 2, \quad 0 \leq \chi \leq 1,
$$

and observe that these functions belong to $C_{c}^{\infty}\left(\mathbb{R}^{2}\right)^{k}$ and satisfy

$$
\int_{\mathbb{R}^{n}}\left|\nabla_{x} \chi\left(\frac{\ln |x|}{\ln m}\right)\right|^{2} d x \leq \frac{2 \pi c_{\chi}}{|\ln m|^{2}} \int_{m^{1 / 2}}^{m} \frac{1}{r^{2}} r d r=\frac{C}{\ln m} .
$$

The nonconstant polynomials lie outside $\mathcal{H}$ because the integral on the left in (3.13) diverges.

So, for $n \geq 3$, formulas (3.7), (3.11) show that the left-hand side of (3.2) can be taken as a scalar product in the Hilbert space $\mathcal{H}$. Furthermore, conditions (3.5) make the righthand side of (3.2) a continuous functional on $\mathcal{H} \ni v$; hence, by the Riesz representation theorem, problem (3.2) has a unique solution, and

$$
\|u ; \mathcal{H}\| \leq c\left(\left\|R f ; L_{2}\left(\mathbb{R}^{n}\right)\right\|+\left\|g ; L_{2}(\Gamma)\right\|\right) .
$$

To verify the claim for $n=2$, observe that, for the same reasons as before and by inequalities (3.7), (3.13), for $\mu>0$, the variational problem

$$
a\left(u, v ; \mathbb{R}^{n}\right)+\mu(u, v)_{\Omega^{0}}=(f, v)_{\mathbb{R}^{n}}+(g, v)_{\Gamma}, \quad v \in \mathcal{H},
$$


is uniquely solvable; also, we have estimate (3.14), in which the weight factor is defined as in the lower formula in (3.4), but the constant $c=c(\mu)$ grows unboundedly as $\mu \rightarrow+0$. It remains to mention that the additional summand $(u, v)_{\Omega^{0}}$ in (3.15) gives rise to a compact perturbation of the operator of problem (3.2) in the space $\mathcal{H}$, and to employ the Fredholm alternative: the orthogonality conditions (3.6) mean that the functional on the right in (3.2) degenerates at the solutions of the homogeneous problem, i.e., at the constant (by the polynomial property (1.8)) columns $b \in \mathbb{C}^{k}$.

We introduce the Kondrat'ev space $V_{\beta}^{l}\left(\Omega^{1}\right)$ (see [24] and also, e.g., the book [25]), depending on the smoothness exponent $l \in \mathbb{N}_{0}:=\{0,1,2, \ldots\}$ and the weight $\beta \in \mathbb{R}$, with the norm

$$
\left\|v ; V_{\beta}^{l}\left(\Omega^{1}\right)\right\|=\left(\sum_{j=0}^{l}\left\||x|^{\beta-l+j} \nabla_{x}^{j} v ; L_{2}\left(\Omega^{1}\right)\right\|^{2}\right)^{1 / 2},
$$

where $\nabla_{x}^{j} v$ is the collection of all derivatives of $v$ of order $j$. The space $V_{\beta}^{l}\left(\Omega^{1}\right)$ is formed by all functions $v \in H_{\mathrm{loc}}^{l}\left(\Omega^{1}\right)$ for which the norm (3.16) is finite. Since the origin of the coordinates $x$ lies off $\overline{\Omega^{1}}$, the weight factors determine the behavior of a function $v \in V_{\beta}^{l}\left(\Omega^{1}\right)$ only at infinity. Therefore, if $l \in \mathbb{N}$, then the trace space $V_{\beta}^{l-1 / 2}(\Gamma)$ coincides with the Sobolev-Slobodetskii space $H^{l-1 / 2}(\Gamma)$ equipped with the norm

$$
\left(\sum_{j=0}^{l-1}\left\|\nabla_{s}^{j} w ; L_{2}(\Gamma)\right\|^{2}+\int_{\Gamma} \int_{\Gamma}\left|\nabla_{s}^{l-1} w(x)-\nabla_{s}^{l-1} w(y)\right|^{2} \frac{d s_{x} d s_{y}}{|x-y|^{n}}\right)^{1 / 2} .
$$

Here $\nabla_{s}$ is the tangent gradient, and $d s_{x}$ is an element of the $(n-1)$-dimensional surface area.

With problem (1.2), (1.3) we associate the mapping

$$
\begin{aligned}
\left\{\mathcal{L}^{0}, \mathcal{L}^{1}, \mathcal{N}^{0}-\mathcal{N}^{1}\right\}: \mathcal{V}_{\beta}^{l+1}\left(\Omega^{0} \cup \Omega^{1}\right)^{k} & \rightarrow \mathcal{R}_{\beta}^{l} V\left(\Omega^{0}, \Omega^{1}, \Gamma\right) \\
& :=H^{l-1}\left(\Omega^{0}\right)^{k} \times V_{\beta}^{l-1}\left(\Omega^{1}\right)^{k} \times H^{l-1 / 2}(\Gamma)^{k},
\end{aligned}
$$

where $\mathcal{V}_{\beta}^{l+1}\left(\Omega^{0} \cup \Omega^{1}\right)$ is the space of functions $v$ such that $v^{0} \in H^{l+1}\left(\Omega^{0}\right), v^{1} \in V_{\beta}^{l+1}\left(\Omega^{1}\right)$, and $v^{0}=v^{1}$ on $\Gamma$. In accordance with the definitions above, the mapping (3.17) is continuous for all indices $l \in \mathbb{N}$ and $\beta \in \mathbb{R}$, but, as is well known, acquires "better properties" only if the weight index lies in a special interval. The general results of [25, $\S 6.1]$ and [3, §2] imply the following statement, based on the polynomial property (1.8).

Proposition 3.2. 1) For $n \geq 3$, The map (3.17) is an isomorphism if and only if $|\beta-l|<-1+n / 2$.

2) Let $n=2$. If $\beta \in(l, l+1)$, the map (3.17) is a Fredholm monomorphism with a $k$-dimensional cokernel formed by the defect functionals indicated on the left-hand side of (3.6), and if $\beta \in(l-1, l)$, then it is a Fredholm epimorphism with a $k$-dimensional kernel spanned by the constant vector-valued functions.

Remark 3.1. Proposition 3.2 is in agreement with Proposition 3.1 and can be used to improve the smoothness of the weak solution. For example, suppose that

$$
(f, g) \in \mathcal{R}_{\beta}^{l} V\left(\Omega^{0}, \Omega^{1}, \Gamma\right), \quad \beta \in \begin{cases}{[l, l-1+n / 2)} & \text { if } n>2, \\ (l, l+1) & \text { if } n=2 .\end{cases}
$$

Then for $n \geq 3$ the requirement (3.5) is fulfilled, and the weak solution $u \in \mathcal{H}$ of problem (1.2), (1.3) becomes classical, belongs to the space $\mathcal{V}_{\beta}^{l+1}\left(\Omega^{0} \cup \Omega^{1}\right)^{k}$, and satisfies

$$
\left\|u ; \mathcal{V}_{\beta}^{l+1}\left(\Omega^{0} \cup \Omega^{1}\right)\right\| \leq c\left\|(f, g) ; \mathcal{R}_{\beta}^{l} V\left(\Omega^{0}, \Omega^{1}, \Gamma\right)\right\| .
$$


Alternatively, if $n=2$, then formulas (3.18) ensure the inequality $\beta>l$, and thereby, the validity of (3.5) with the weight factor (3.4). Thus, by Proposition 3.1 if the solvability conditions are satisfied, then problem (1.2), (1.3) admits a weak solution $u \in \mathcal{H}$ determined up to a constant summand. At the same time, Proposition 3.2(2) (see the description of the cokernel) provides a unique classical solution $u \in \mathcal{V}_{\beta}^{l+1}\left(\Omega^{0} \cup \Omega^{1}\right)^{k} \cap \mathcal{H}$ and guarantees estimate (3.19). The criterion for choosing a unique solution is explained in Remark 3.2 .

The theorem on asymptotics for solutions of exterior boundary-value problems (see [24, 26] and also [25, §6.4]) yields a representation of the solutions of the transmission problem (1.2), (1.3) as $r=|x| \rightarrow \infty$. Let $\Phi(x)$ denote the fundamental matrix for the operator $\mathcal{L}^{1}\left(\nabla_{x}\right)$ in the space $\mathbb{R}^{n}$, which satisfies the relation

$$
\mathcal{L}^{1}\left(\nabla_{x}\right) \Phi(x)=\mathbb{I}_{k} \delta(x), \quad x \in \mathbb{R}^{n},
$$

where $\delta$ is the Dirac function, and $\mathbb{I}_{k}$ is the unit matrix of size $k \times k$.

It is known (see, e.g., [27]) that

$$
\Phi(x)= \begin{cases}r^{2-n} \Phi^{0}(\varphi) & \text { if } n>2, \\ C_{\Phi} \ln r+\Phi^{0}(\varphi) & \text { if } n=2,\end{cases}
$$

where $(r, \varphi)$ are the spherical coordinates, $\Phi^{0}$ is a smooth matrix-valued function on the unit sphere $\mathbb{S}^{n-1}=\partial \mathbb{B}_{1}^{n}$, and $C_{\Phi}$ is a constant $(k \times k)$-matrix; if $n=2$, then the entries of $\Phi^{0}$ have zero mean. Identity (3.20) can be understood as follows:

$$
\int_{\mathbb{S}_{R}^{n-1}} \mathcal{N}^{1}\left(x, \nabla_{x}\right) \Phi(x) d s_{x}=\int_{\mathbb{S}_{R}^{n-1}} \overline{\mathcal{D}^{\top}\left(\frac{x}{R}\right)} \mathcal{A}^{1} \mathcal{D}\left(\nabla_{x}\right) \Phi(x) d s_{x}=\mathbb{I}_{k} .
$$

Note that $R^{-1} x$ is the outward unit normal to the sphere $\mathbb{S}_{R}^{n-1}=\{x: r=R\}$, and that the two integrals in (3.22) do not depend on the radius $R>0$ of the sphere.

Proposition 3.3. Suppose that

$$
(f, g) \in \mathcal{R}_{\gamma}^{l} V\left(\Omega^{0}, \Omega^{1}, \Gamma\right), \quad \gamma \in(l+n / 2, l+1+n / 2),
$$

which ensures, in particular, the membership relation in (3.18); if $n=2$, we assume additionally the orthogonality condition (3.6). Then the solution $u \in \mathcal{V}_{\beta}^{l+1}\left(\Omega^{0} \cup \Omega^{1}\right)^{k}$ of problem (1.2), (1.3), as presented in Proposition 3.2, admits the expansion

$$
u^{1}(x)=\sum_{p=1}^{k} c_{0, p} \Phi^{p}(x)+\sum_{p=1}^{k} \sum_{j=1}^{n} c_{j, p} \frac{\partial \Phi^{p}}{\partial x_{j}}(x)+\widetilde{u}^{1}(x),
$$

where the $\Phi^{p}(x)$ are the columns of the fundamental matrix $\Phi(x)$, the remainder term $\widetilde{u}^{1}$ belongs to $V_{\gamma}^{l+1}\left(\Omega^{1}\right)^{k}$, and the $c_{j, p}$ are some constants; in the case $n=2$ we have

$$
c_{0,1}=\cdots=c_{0, k}=0 .
$$

Also, the following estimate is valid:

$$
\sum_{p=0}^{k} \sum_{j=1}^{n}\left|c_{j, p}\right|+\left\|\widetilde{u}^{1} ; V_{\gamma}^{l+1}\left(\Omega^{1}\right)\right\| \leq c\left\|(f, g) ; \mathcal{R}_{\gamma}^{l} V\left(\Omega^{0}, \Omega^{1}, \Gamma\right)\right\| .
$$

Remark 3.2. If $n=2$ and $u \in \mathcal{V}_{\beta}^{l+1}\left(\Omega^{0} \cup \Omega^{1}\right)^{k}$ is the solution of (1.2), (1.3) indicated in Remark 3.1 then $u+c$ is a weak solution belonging to the energy class $\mathcal{H}$ for any column $c \in \mathbb{C}^{k}$, because $c \in \mathcal{H}$ (this was proved before), and $\mathcal{V}_{\beta}^{l+1}\left(\Omega^{0} \cup \Omega^{1}\right)^{k} \subset \mathcal{H}$ by the definitions of the norms (3.3), (3.16) and the restriction (3.18) imposed on the weight index. In other words, the solutions mentioned in Propositions 3.1 and 3.2 differ by a constant summand. In Proposition 3.3, this fact was taken into account by identity 
(3.25), but the corresponding statement can easily be adjusted to weak solutions: it suffices to remove identity (3.25) and delete the index $p=0$ in summation in (3.26).

Throughout in what follows, if the smoothness exponent $l \in \mathbb{N}$ is fixed, then the weight indices $\beta$ and $\gamma$ are assumed to satisfy the restrictions mentioned in (3.18) and (3.23).

\section{$\S 4$. The polarization matrix}

We start with writing the differential operators $\mathcal{L}^{i}$ in a simpler form. Permuting the rows of the matrix $\mathcal{D}(\xi)$, we can arrange that the top $N_{0}$ rows be linearly independent and the bottom $N_{1}=N-N_{0}$ rows be linear combinations of the top rows. Of course, this permutation is accompanied by the corresponding reconstruction of the matrices $\mathcal{A}^{i}$ and the columns $u^{i}$ and $f^{i}, g$. As a result, we arrive at the formulas

$$
\mathcal{A}^{i}=\left(\begin{array}{ll}
\mathcal{A}_{(00)}^{i} & \mathcal{A}_{(01)}^{i} \\
\mathcal{A}_{(10)}^{i} & \mathcal{A}_{(11)}^{i}
\end{array}\right), \quad \mathcal{D}\left(\nabla_{x}\right)=\left(\begin{array}{c}
\mathcal{D}_{(0)}\left(\nabla_{x}\right) \\
\mathcal{T}_{(0)}\left(\nabla_{x}\right)
\end{array}\right)
$$

where the $\mathcal{A}_{(00)}^{i}$ and $\mathcal{A}_{(11)}^{i}$ are Hermitian positive definite matrices of size $N_{0} \times N_{0}$ and $N_{1} \times N_{1}$, respectively, and the matrices $\mathcal{A}_{(10)}^{i}=\left(\overline{\mathcal{A}_{(01)}^{i}}\right)^{\top}$ and $\mathcal{T}$ are of size $N_{1} \times N_{0}$. By (1.1) and (4.1), we have

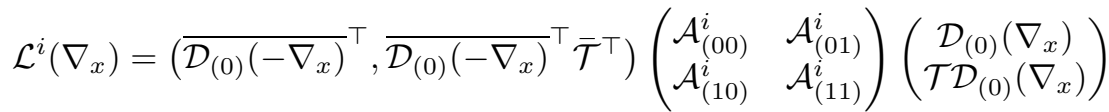

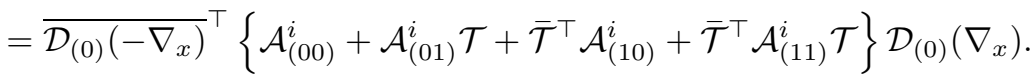

Here, in braces we have an $\left(N_{0} \times N_{0}\right)$-matrix $\mathcal{A}_{(\mathcal{T})}^{i}$, which is Hermitian for obvious reasons and positive definite because

$$
\bar{\xi}^{\top} \mathcal{A}_{(\mathcal{T})}^{i} \xi=\left(\bar{\xi}^{\top}, \bar{\xi}^{\top} \overline{\mathcal{T}}^{\top}\right)\left(\begin{array}{ll}
\mathcal{A}_{(00)}^{i} & \mathcal{A}_{(01)}^{i} \\
\mathcal{A}_{(10)}^{i} & \mathcal{A}_{(11)}^{i}
\end{array}\right)\left(\begin{array}{c}
\xi \\
\mathcal{T} \xi
\end{array}\right)>0, \quad \xi \in \mathbb{C}^{N_{0}} \backslash\{0\} .
$$

Thus, the operators (1.1) and (1.4), and also the forms (1.7), admit a concise writing with matrices $\mathcal{A}^{i}$ and $\mathcal{D}$ of smaller size. We assume that such simplifications have been done from the outset, and thereby, since the rows of $\mathcal{D}(\xi)$ are linearly independent,

$$
\mathcal{D}\left(\nabla_{x}\right) \mathbf{L}^{1}=\mathbb{C}^{N}
$$

where $\mathbf{L}^{1}$ is the linear space of homogeneous linear vector-valued functions

$$
l(x)=\left(l_{1}(x), \ldots, l_{k}(x)\right)^{\top}, \quad l_{r}(x)=l_{r}^{1} x_{1}+\cdots+l_{r}^{n} x_{n}, \quad r=1, \ldots, k .
$$

We identify this linear space with $\mathbb{C}^{k \times n}$ by reducing the functions (4.2) to the coefficient columns $\left(l_{1}^{1}, \ldots, l_{1}^{k}, l_{2}^{1}, \ldots, l_{2}^{k}, \ldots, l_{k}^{1}, \ldots, l_{k}^{k}\right)^{\top}$. We have

$$
\sum_{r=1}^{k} \overline{m_{r}\left(\nabla_{x}\right)} l_{r}(x)=\sum_{r=1}^{k} \sum_{h=1}^{n} \overline{m_{r}^{h}} l_{r}^{h}
$$

The right-hand side is a scalar product in $\mathbb{C}^{k \times n}$; hence, the form on the left in (4.3) induces a scalar product in the Hilbert space $\mathbf{L}^{1}$ of the vector polynomials (4.2).

We denote by $\overline{\mathcal{D}}^{1}(x), \ldots, \overline{\mathcal{D}}^{N}(x)$ the columns of the $(k \times N)$-matrix $\overline{\mathcal{D}(x)}^{\top}$, belonging to $\mathbf{L}^{1}$. The Gram matrix

$$
\mathbf{D}=\mathcal{D}\left(\nabla_{x}\right)\left(\overline{\mathcal{D}}^{1}(x), \ldots, \overline{\mathcal{D}}^{N}(x)\right)=\mathcal{D}\left(\nabla_{x}\right) \overline{\mathcal{D}(x)}^{\top} \in \mathbb{C}^{N \times N}
$$

is Hermitian and positive definite. Let $\mathbf{D}^{1 / 2}$ be the positive square root of $\mathbf{D}$. We modify 
the writing of the operators $\mathcal{L}^{i}$ once again:

$$
\mathcal{L}^{i}\left(\nabla_{x}\right)={\overline{\mathbf{D}^{-1 / 2}} \mathcal{D}\left(-\nabla_{x}\right)}^{\top}\left\{\left(\overline{\mathbf{D}}^{1 / 2}\right)^{\top} \mathcal{A}^{i} \mathbf{D}^{1 / 2}\right\} \mathbf{D}^{-1 / 2} \mathcal{D}\left(\nabla_{x}\right) .
$$

The matrices $\widetilde{\mathcal{A}}^{i}=\left(\overline{\mathbf{D}}^{1 / 2}\right)^{\top} \mathcal{A}^{i} \mathbf{D}^{1 / 2}$ and $\widetilde{\mathcal{D}}\left(\nabla_{x}\right)=\mathbf{D}^{-1 / 2} \mathcal{D}\left(\nabla_{x}\right)$ keep all the properties of $\mathcal{A}^{i}$ and $\mathcal{D}\left(\nabla_{x}\right)$ assumed above. Moreover, we have

$$
\widetilde{\mathcal{D}}\left(\nabla_{x}\right) \overline{\widetilde{\mathcal{D}}}(x)^{\top}=\mathbf{D}^{-1 / 2} \mathcal{D}\left(\nabla_{x}\right) \overline{\mathcal{D}(x)}^{\top}\left(\overline{\mathbf{D}}^{\top}\right)^{-1 / 2}=\mathbf{D}^{-1 / 2} \mathbf{D D}^{-1 / 2}=\mathbb{I}_{N} .
$$

Again, we assume that the changes $\mathcal{A}^{i} \mapsto \widetilde{\mathcal{A}}^{i}$ and $\mathcal{D}\left(\nabla_{x}\right) \mapsto \widetilde{\mathcal{D}}\left(\nabla_{x}\right)$ have been made from the outset. As a result, after a due redenotation, we arrive at the identity

$$
\mathcal{D}\left(\nabla_{x}\right) \overline{\mathcal{D}(x)}^{\top}=\mathbb{I}_{N} \in \mathbb{C}^{N \times N},
$$

which is important for what follows, while the operators (1.1), (1.4) and the forms (1.7) remain the same.

We fix a specific basis in the linear space $\mathbf{L}=\mathbf{L}^{1} \cup \mathbf{L}^{0}$ of linear vector-valued functions:

$$
\underbrace{q^{1}, \ldots, q^{N}}, \quad \underbrace{q^{N+1}, \ldots, q^{k n}}, \quad \underbrace{q^{k n+1}, \ldots, q^{k(n+1)}} .
$$

The first $N$ and the last $k$ elements are given by

$$
\begin{aligned}
q^{j}(x) & =\overline{\mathcal{D}}^{j}(x), \quad j=1, \ldots, N, \\
q^{k n+p}(x) & =e_{(p)}:=\left(\delta_{1, p}, \ldots, \delta_{k, p}\right)^{\top}, \quad p=1, \ldots, k .
\end{aligned}
$$

The remaining elements form a basis in the linear space $\mathbf{L}^{1} \cap\left(\mathcal{P} \ominus \mathbb{C}^{k}\right)$ (recall the polynomial property (1.9) and the scalar product (4.3) in the space $\mathbf{L}^{1}$ ). They are chosen arbitrarily, but all the vector-valued functions in (4.5) are subject to the conditions

$$
\left.\sum_{r=1}^{k} \overline{q_{r}^{j}\left(\nabla_{x}\right)} q_{r}^{p}(x)\right|_{x=0}=\delta_{j, p} .
$$

It should be mentioned that for the vectors (4.2) the substitution symbol $\left.\right|_{x=0}$ in formula (4.7) is not needed, but its presence makes it possible to extend this formula to all indices $j, p=1, \ldots, k(n+1)$.

The linear hulls of the vectors $q^{1}, \ldots, q^{N}$ and $q^{N+1}, \ldots, q^{k(n+1)}$ are denoted by $\mathbf{L}_{\mathcal{D}}$ and $\mathbf{L}_{d}$, respectively.

Remark 4.1. 1) For the scalar operator of Example 1.1 we have $\mathbf{L}_{d}=\mathbb{C}$ and $\mathbf{L}_{\mathcal{D}}=$ $\left\{a_{1} x_{1}+\cdots+a_{n} x_{n}: a_{j} \in \mathbb{C}\right\}$. The requirement (4.4) is fulfilled.

2) In the case of the 3-dimensional system of elasticity theory (see Example 1.2),

$$
\mathbf{L}_{d}=\left\{d(x) a: a \in \mathbb{R}^{6}\right\}, \quad \mathbf{L}_{\mathcal{D}}=\left\{\mathcal{D}(x)^{\top} b: b \in \mathbb{R}^{6}\right\},
$$

where $d$ and $\mathcal{D}^{\top}$ are the matrices (1.10) and (1.11). In the 2-dimensional case (see Example 1.3), the two linear spaces (4.8) become 3-dimensional, but they are given by the same formulas as before with the matrices $d$ and $\mathcal{D}^{\top}$ as in (1.13) and (1.14). The normalization conditions (4.4) are satisfied due to the factor $2^{-1 / 2}$ in the formula for $\mathcal{D}$.

The vectors $q^{N+1}, \ldots, q^{k(n+1)}$ are solutions of the homogeneous $(f=0$ and $g=0)$ problem (1.2), (1.3), because $q^{j} \in \mathcal{P}$ and $\mathcal{D}\left(\nabla_{x}\right) q^{j}(x)=0$ for $j=N+1, \ldots, k(n+1)$. On the other hand, the vectors $q^{1}, \ldots, q^{N}$ leave discrepancies in the second transmission condition (1.3), but satisfy (1.2). For $j=1, \ldots, N$, there exists a solution $\widehat{\zeta}^{(j)} \in$ $\mathcal{V}_{\beta}^{l+1}\left(\Omega^{0} \cup \Omega^{1}\right)^{k}$ of problem (1.2), (1.3) with the right-hand sides

$$
f^{(j) 0}=0, \quad f^{(j) 1}=0, \quad g^{(j)}=\overline{\mathcal{D}(\nu)}^{\top}\left(\mathcal{A}^{1}-\mathcal{A}^{0}\right) \mathcal{D}\left(\nabla_{x}\right) q^{j} \in C^{\infty}(\Gamma)^{k} .
$$


Note that $\mathcal{D}\left(\nabla_{x}\right) q^{j}$ is a constant column of height $N$, and the orthogonality conditions (3.6) are fulfilled for $n=2$ because $\int_{\Gamma} \nu(x) d s_{x}=0$. Thus, the sums $\zeta^{(j)}=q^{j}+\widehat{\zeta}^{(j)}$ also turn out to be solutions of the homogeneous problem (1.2), (1.3) with linear growth at infinity.

Since the right-hand sides (4.9) satisfy (3.23), the vector-valued functions $\hat{\zeta}^{(j)}, j=$ $1, \ldots, N$, admit the expansions (3.24), which we rewrite as follows:

$$
\widehat{\zeta}^{(j) 1}(x)=\sum_{p=N+1}^{k(n+1)} \sum_{r=1}^{k} C_{j p} q_{r}^{p}\left(-\nabla_{x}\right) \Phi^{r}(x)-\sum_{p=1}^{N} \sum_{r=1}^{k} P_{j p} q_{r}^{p}\left(-\nabla_{x}\right) \Phi^{r}(x)+\tilde{\zeta}^{j 1}(x) .
$$

Our nearest goal is to check that, for the specific right-hand sides (4.9), we have

$$
\begin{aligned}
C_{j p} & =0, \quad j=1, \ldots, N, \quad p=N+1, \ldots, k(n+1), \\
P_{j p} & =\overline{P_{p j}}, \quad j, p=1, \ldots, N .
\end{aligned}
$$

We use the method of 28 .

Here we deal with the polarization matrix $P$, mentioned in $\S 2$ and composed of the coefficients $P_{j p}$ in the expansions (4.10).

Proposition 4.1. Identities (4.11) and (4.12) are valid; in particular, the $(N \times N)$ matrix $P$ is Hermitian. If the eigenvalues of the $(N \times N)$-matrix $\mathcal{A}^{1}-\mathcal{A}^{0}$ are nonnegative, i.e., $\mathcal{A}^{1} \geq \mathcal{A}^{0}$, then the polarization matrix $P$ is negative definite.

Proof. We plug the fields $q^{p}, p=N+1, \ldots, k(n+1)$, and $\widehat{\zeta}^{(j)}, j=1, \ldots, N$, in the Green formula (3.1) for the composite body $\Omega_{R}^{1} \cup \Omega^{0}$, where $\Omega_{R}^{1}=\left\{x \in \Omega^{1}:|x|<R\right\}$ is the intersection of the domain $\Omega^{1}$ and the ball $\mathbb{B}_{R}=\{x:|x|<R\}$ of a large radius $R$. We have

$$
\begin{aligned}
0 & =\int_{\Omega^{0}}\left(\overline{\mathcal{D}\left(\nabla_{x}\right) q^{p}(x)}\right)^{\top}\left(\mathcal{A}^{1}-\mathcal{A}^{0}\right) \mathcal{D}\left(\nabla_{x}\right) q^{j}(x) d x \\
& \left.=\int_{\Gamma}{\overline{q^{p}(x)}}^{\top} \overline{\mathcal{D}(\nu(x))}\right)^{\top}\left(\mathcal{A}^{1}-\mathcal{A}^{0}\right) \mathcal{D}\left(\nabla_{x}\right) q^{j}(x) d s_{x} \\
& =\int_{\Gamma}{\overline{q^{p}(x)}}^{\top} \overline{\mathcal{D}(\nu(x))}{ }^{\top}\left(\mathcal{A}^{0} \mathcal{D}\left(\nabla_{x}\right) \hat{\zeta}^{(j) 0}(x)-\mathcal{A}^{1} \mathcal{D}\left(\nabla_{x}\right) \hat{\zeta}^{(j) 1}(x)\right) d s_{x} \\
& \left.=\int_{\mathbb{S}_{R}^{n-1}}{\overline{q^{p}(x)}}^{\top} \overline{\mathcal{D}\left(R^{-1} x\right)}\right)^{\top} \mathcal{A}^{1} \mathcal{D}\left(\nabla_{x}\right) \hat{\zeta}^{(j) 1}(x) d s_{x} \\
& =\int_{\mathbb{S}_{R}^{n-1}}{\overline{q^{p}(x)}}^{\top}{\overline{\mathcal{D}\left(R^{-1} x\right)}}^{\top} \mathcal{A}^{1} \mathcal{D}\left(\nabla_{x}\right) \Sigma^{(j)}(x) d s_{x}+o(1) \\
& =\int_{\mathbb{B}_{R}^{n}}{\overline{q^{p}(x)}}^{\top} \overline{\mathcal{D}\left(\nabla_{x}\right)}{ }^{\top} \mathcal{A}^{1} \mathcal{D}\left(\nabla_{x}\right) \Sigma^{(j)}(x) d s_{x}+o(1) \\
& =\int_{\mathbb{B}_{R}^{n}}{\overline{q^{p}(x)}}^{\top}\left(\sum_{h=J+1}^{k(n+1)} C_{j h} q^{h}\left(-\nabla_{x}\right) \delta(x)-\sum_{h=1}^{J} P_{j h} q^{h}\left(-\nabla_{x}\right) \delta(x)\right) d x+o(1) \\
& =\left.\sum_{r=1}^{k}\left(\sum_{h=J+1}^{k(n+1)} C_{j h} q_{r}^{h}\left(\nabla_{x}\right) \overline{q_{r}^{p}(x)}-\sum_{h=1}^{J} P_{j h} q_{r}^{h}\left(\nabla_{x}\right) \overline{q_{r}^{p}(x)}\right)\right|_{x=0}+o(1) \\
& =C_{j p}+o(1) .
\end{aligned}
$$

Some comments on the above calculation are in order. First, we used the fact that $\mathcal{D}\left(\nabla_{x}\right) q^{p}=0$ and the Gauss-Ostrogradskiu formula, together with the expression (4.9) for the right-hand side $g^{j}$ of the second transmission condition (1.3) for $\widehat{\zeta}^{(j)}$. Then we used the Green formula mentioned above: the volume integrals disappeared by the first 
two identities in (4.9), and, in the integral over the sphere $\mathbb{S}_{R}^{n-1}$ with the unit normal $R^{-1} x$, the vector-valued function $\widehat{\zeta}^{(j)}$ is replaced with the sum $\Sigma^{(j)}$ of asymptotic terms singled out in (4.10). The impact of the remainder term $\widetilde{\zeta}^{(j)} \in V_{\gamma}^{l+1}\left(\Omega^{1}\right)^{k}$ is estimated as follows:

$$
\begin{aligned}
& \int_{\mathbb{S}_{R}^{n-1}}|x|\left|\nabla_{x} \widetilde{\zeta}^{(j) 1}(x)\right| d s_{x} \leq c R^{(n-1) / 2} R\left(\int_{\mathbb{S}_{R}^{n-1}}\left|\nabla_{x} \widetilde{\zeta}^{(j) 1}(x)\right|^{2} d s_{x}\right)^{1 / 2} \\
& \leq c R^{(n+1) / 2} R^{1 / 2}\left(\int_{\mathbb{B}_{R}^{n} \backslash \mathbb{B}_{R / 2}^{n}}\left(\left|\nabla_{x}^{2} \widetilde{\zeta}^{(j) 1}(x)\right|^{2}+R^{-2}\left|\nabla_{x} \widetilde{\zeta}^{(j) 1}(x)\right|^{2}\right) d x\right)^{1 / 2} \\
& \leq c R^{1+n / 2} R^{-(\gamma-l-1+2)} \\
& \quad \times\left(\int_{\Omega^{1}}\left(|x|^{2(\gamma-l-1+2)}\left|\nabla_{x}^{2} \widetilde{\zeta}^{(j) 1}(x)\right|^{2}+|x|^{2(\gamma-l-1+1)}\left|\nabla_{x} \widetilde{\zeta}^{(j) 1}(x)\right|^{2}\right) d x\right)^{1 / 2} \\
& \leq c R^{l-\gamma+n / 2}\left\|\widetilde{\zeta}^{(j) 1} ; V_{\gamma}^{l+1}\left(\Omega^{1}\right)\right\| .
\end{aligned}
$$

Here we have used the embedding $H^{1}\left(\mathbb{B}_{1}^{n} \backslash \mathbb{B}_{1 / 2}^{n}\right) \subset L_{2}\left(\mathbb{S}_{1}^{n-1}\right)$ (after the coordinate dilation $x \mapsto \mathbf{x}=R^{-1} x$ ) and placed the weight factors under the integral, due to the relation $R>|x| \geq R / 2, x \in \mathbb{B}_{1}^{n} \backslash \mathbb{B}_{1 / 2}^{n}$ (cf. the weight norm (3.16)). The coefficient of the last-written norm is infinitesimally small as $R \rightarrow \infty$ because $\gamma>l+n / 2$ (see the restriction (3.23)). Finally, we understand the integral over the ball $\mathbb{B}_{R}$ in the distributional sense, applying equation (3.20) for the fundamental matrix $\Phi$, and also the normalization conditions (4.7) for the polynomial basis (4.5) (an alternative approach to the calculation of the integral over the sphere $\mathbb{S}_{R}^{n-1}$ in (4.13) is based on relation (3.22)).

Thus, identity (4.11) is deduced from (4.13) by the limit passage as $R \rightarrow+\infty$.

Similar transformations can be done after plugging the vector-valued functions $\zeta^{(p)}=$ $q^{p}+\widehat{\zeta}^{(p)}$ and $\widehat{\zeta}^{(j)}, j, p=1, \ldots, N$ in the Green formula. Taking the transmission conditions for $\hat{\zeta}^{(p)}$ into account, as a result we find

$$
\begin{aligned}
& \int_{\Gamma}{\overline{\zeta^{(p)}(x)}}^{\top} \overline{\mathcal{D}(\nu(x))}{ }^{\top}\left(\mathcal{A}^{0} \mathcal{D}\left(\nabla_{x}\right) \hat{\zeta}^{(j) 0}(x)-\mathcal{A}^{1} \mathcal{D}\left(\nabla_{x}\right) \hat{\zeta}^{(j) 1}(x)\right) d s_{x} \\
& \quad=\left.\sum_{r=1}^{k}\left(\sum_{h=J+1}^{k(n+1)} C_{j h} q_{r}^{h}\left(\nabla_{x}\right) \overline{q_{r}^{p}(x)}-\sum_{h=1}^{J} P_{j h} q_{r}^{h}\left(\nabla_{x}\right) \overline{q_{r}^{p}(x)}\right)\right|_{x=0}+o(1) \\
& =-P_{j p}+o(1) .
\end{aligned}
$$

The quantity $o(1)$ is removed by the limit passage as $R \rightarrow+\infty$. On the other hand, we have

$$
\begin{aligned}
-P_{j p}= & \int_{\Gamma}{\overline{\hat{\zeta}^{(p)}(x)}}^{\top} \overline{\mathcal{D}(\nu(x))}^{\top}\left(\mathcal{A}^{0} \mathcal{D}\left(\nabla_{x}\right) \widehat{\zeta}^{(j) 0}(x)-\mathcal{A}^{1} \mathcal{D}\left(\nabla_{x}\right) \hat{\zeta}^{(j) 1}(x)\right) d s_{x} \\
& -\int_{\Gamma}{\overline{q^{p}(x)}}^{\top} \overline{\mathcal{D}(\nu(x))}^{\top}\left(\mathcal{A}^{0}-\mathcal{A}^{1}\right) \mathcal{D}\left(\nabla_{x}\right) q^{j}(x) d s_{x} \\
= & a\left(\hat{\zeta}^{(j)}, \hat{\zeta}^{(p)} ; \mathbb{R}^{n}\right)+\left(\left(\mathcal{A}^{1}-\mathcal{A}^{0}\right) \mathcal{D}\left(\nabla_{x}\right) q^{j}, \mathcal{D}\left(\nabla_{x}\right) q^{p}\right)_{\Omega^{0}} .
\end{aligned}
$$

The right-hand side is the sum of two Hermitian forms evaluated at the pairs $\widehat{\zeta}^{(j)}, \widehat{\zeta}^{(p)}$ and $q^{j}, q^{p}$, i.e., $P$ is a Hermitian matrix. Moreover, the last but one elements in (4.16) form a Gram matrix, which is positive definite, because the solutions $\widehat{\zeta}^{(1)}, \ldots, \widehat{\zeta}^{(N)}$ are linearly independent and the form $a\left(\cdot, \cdot ; \mathbb{R}^{n}\right)$ is a scalar product in the energy space $\mathcal{H}$ for $n \geq 3$, while for $n=2$ this form is such in the factor space $\mathcal{H} / \mathbb{C}^{n}$, to which all the solutions mentioned belong (see Remark 3.2). Finally, by (4.5) and (4.4), the last term in (4.16) is equal to $\left(\mathcal{A}_{j p}^{1}-\mathcal{A}_{j p}^{0}\right)$ meas $_{n} \Omega^{0}$, which makes the second statement of Proposition 4.1 evident. 
Remark 4.2. Proposition 4.1 remains valid also in the case where $\mathcal{A}^{0}=\mathbb{O}$, i.e., for the cavity $\Omega^{0}$. In that case, the interior of the set $\overline{\Omega^{0}}$ may be empty; for instance, this set can be part of an $(n-1)$-dimensional surface on the sides of which the Neumann boundary conditions can be posed. Since meas $\bar{\Omega}^{0}=0$ and the last term in (4.16) vanishes, the matrix $P$ can lose its negative definiteness, but will be singular negative for sure. In the exterior elasticity theory problem, the polarization matrix of size $3 \times 3$ for a crack that is a segment on the elastic plane $\mathbb{R}^{2}$ has rank 2 , and the polarization matrix of size $6 \times 6$ for a plane crack in the elastic space $\mathbb{R}^{3}$ has rank 3 (see [7]). At the same time, for curved cracks the polarization matrices may remain nonsingular.

Deepening the matrix form of writing, we put

$$
\zeta(x)=\left(\zeta^{(1)}(x), \ldots, \zeta^{(N)}(x)\right)=\overline{\mathcal{D}(x)}^{\top}+\widehat{\zeta}(x),
$$

where $\hat{\zeta}=\left(\widehat{\zeta}^{(1)}, \ldots, \hat{\zeta}^{(N)}\right) \in \mathcal{V}_{\beta}^{l+1}\left(\Omega^{1} \cup \Omega^{0}\right)^{k \times N}$ is the $(k \times N)$-matrix whose columns are solutions of problem (1.2), (1.3) with the right-hand sides (4.9). Using the identities (4.10) (already checked), we see that the expansions (4.11) read

$$
\widehat{\zeta}^{1}(x)=\left(\overline{\mathcal{D}\left(\nabla_{x}\right)} \Phi(x)^{\top}\right)^{\top} P+\widetilde{\zeta}^{1}(x), \quad \widetilde{\zeta}^{1} \in V_{\gamma}^{l+1}\left(\Omega^{1}\right)^{k \times N} .
$$

Note that the double transposition is introduced to match the order of differentiation and multiplication of matrices; the weight indices $\beta$ and $\gamma$ are taken from (3.18) and (3.23), respectively. In the matrix form, the representation (4.16) reads

$$
P=-a\left(\widehat{\zeta}, \widehat{\zeta} ; \mathbb{R}^{n}\right)-\left(\mathcal{A}^{1}-\mathcal{A}^{0}\right) \operatorname{meas}_{n} \Omega^{0} .
$$

Remark 4.3. Since the system (1.2) of differential equations for the matrix-valued functions $\zeta$ in $\Omega^{1}$ is homogeneous, the results of [29] provide estimates for the asymptotic remainder terms at infinity. For the entries of $\zeta$ itself this yields

$$
\begin{aligned}
& \left|\nabla_{x}^{j} \zeta_{p q}(x)\right| \leq c_{j}|x|^{1-j}, \quad j=0,1, \\
& \left|\nabla_{x}^{j} \zeta_{p q}(x)\right| \leq c_{j}|x|^{1-n-j}, \quad j \in \mathbb{N}, \quad j>1, \quad x \in \mathbb{R}^{n},
\end{aligned}
$$

and for the components $\widehat{\zeta}$ and $\tilde{\zeta}$ we have

$$
\left|\nabla_{x}^{j} \widehat{\zeta}_{p q}(x)\right| \leq c_{j}|x|^{1-n-j}, \quad\left|\nabla_{x}^{j} \widetilde{\zeta}_{p q}(x)\right| \leq c_{j}|x|^{-n-j}, \quad j \in \mathbb{N}_{0}, \quad x \in \mathbb{R}^{n}
$$

Formulas (4.20) reflect the fact that $\zeta(x)$ grows linearly as $|x| \rightarrow \infty$. Observe that a faster rate of decay of the entries of $\widehat{\zeta}$ is due to the relations $C_{j, k n+1}=\cdots=C_{j, k(n+1)}=0$ (see (4.11) and (4.5), (4.6)). In general, the solution $u$ of problem (1.2), (1.3) with the right-hand side $f^{1}=0$ is subject to the estimates

$$
\left|\nabla_{x}^{j} u^{1}(x)\right| \leq c_{j}|x|^{2-n-j}, \quad x \in \mathbb{R}^{n}, \quad j \in \mathbb{N}_{0} ;
$$

for $n=2$ and $j=0$ the majorant $c_{0}$ in (4.21) should be replaced with $c_{0}(1+|\ln | x||)$ in accordance with (3.21) and (3.24).

Proposition 4.2. If the eigenvalues of the $(N \times N)$-matrix $\left(\mathcal{A}^{1}\right)^{-1}-\left(\mathcal{A}^{0}\right)^{-1}$ are nonnegative, i.e., $\left(\mathcal{A}^{1}\right)^{-1} \geq\left(\mathcal{A}^{0}\right)^{-1}$, then the polarization matrix $P$ is positive definite.

Proof. We represent the matrix of special solutions (4.17) in the form

$$
\zeta(x)= \begin{cases}\overline{\mathcal{D}(x)}^{\top}+\hat{z}^{1}(x) & \text { for } x \in \Omega^{1}, \\ \overline{\mathcal{D}(x)}^{\top}\left(\mathcal{A}^{0}\right)^{-1} \mathcal{A}^{1}+\hat{z}^{0}(x) & \text { for } x \in \Omega^{0} .\end{cases}
$$

Clearly, $\widehat{z}^{1}=\widehat{\zeta}^{1}$, but $\widehat{z}^{0}$ differs from $\widehat{\zeta}^{0}$; in particular, the second transmission conditions (1.3) for the matrix-valued function $\widehat{z}=\left\{\hat{z}^{1}, \widehat{z}^{0}\right\}$ become homogeneous, because

$$
\overline{\mathcal{D}(\nu(x))}^{\top} \mathcal{A}^{0} \mathcal{D}\left(\nabla_{x}\right) \overline{\mathcal{D}(x)}^{\top}\left(\mathcal{A}^{0}\right)^{-1} \mathcal{A}^{1}=\overline{\mathcal{D}(\nu(x))}^{\top} \mathcal{A}^{1},
$$


which is ensured by the normalization (4.4). The representation (4.22) implies the transmission condition

$$
\widehat{z}^{0}(x)-\widehat{z}^{1}(x)=\overline{\mathcal{D}(x)}^{\top}\left(\mathbb{I}_{N}-\left(\mathcal{A}^{0}\right)^{-1} \mathcal{A}^{1}\right) .
$$

Here $\widehat{z}^{0}(x)=\widehat{\zeta}^{0}(x)+\overline{\mathcal{D}(x)}^{\top}\left(\mathbb{I}_{N}-\left(\mathcal{A}^{0}\right)^{-1} \mathcal{A}^{1}\right)$.

Repeating the calculations in the proof of Proposition 4.1, this time with the matrixvalued functions $\zeta$ and $\widehat{z}$, we arrive at the formula

$$
-P=-\int_{\Gamma}\left({\overline{\widehat{z}^{0}(x)-\hat{z}^{1}(x)}}^{\top} \overline{\mathcal{D}}(\nu(x))^{\top} \mathcal{A}^{i} \mathcal{D}\left(\nabla_{x}\right) \zeta^{i}(x) d x .\right.
$$

It should be noted that, first, the factor in the integrand does not depend on $i=0,1$, and second, the minus sign, absent in (4.15) and (4.16), is determined by the homogeneous first and nonhomogeneous second transmission conditions (1.3) for $\widehat{z}$. Using the definition (4.22) and the transmission conditions (4.23), we deduce the following representation, similar to (4.19):

$$
\begin{aligned}
& P=\int_{\Gamma}\left({\overline{\widehat{z}^{0}(x)-\widehat{z}^{1}(x)}}^{\top} \overline{\mathcal{D}(\nu(x))}^{\top} \mathcal{A}^{i} \mathcal{D}\left(\nabla_{x}\right) \widehat{z}^{i}(x) d x\right. \\
& +\int_{\Gamma}{\overline{\overline{\mathcal{D}(x)}\left(\mathbb{I}_{N}-\left(\mathcal{A}^{0}\right)^{-1} \mathcal{A}^{1}\right)}}^{\top} \overline{\mathcal{D}(\nu(x))}^{\top} \mathcal{A}^{1} \mathcal{D}\left(\nabla_{x}\right) \overline{\mathcal{D}(x)}^{\top} d x \\
& =a\left(\hat{z}, \widehat{z} ; \mathbb{R}^{n}\right)+\int_{\Omega^{0}}\left(\mathbb{I}_{N}-\mathcal{A}^{1}\left(\mathcal{A}^{0}\right)^{-1}\right) \mathcal{A}^{1} d x \\
& =a\left(\widehat{z}, \widehat{z} ; \mathbb{R}^{n}\right)+\mathcal{A}^{1}\left(\left(\mathcal{A}^{1}\right)^{-1}-\left(\mathcal{A}^{0}\right)^{-1}\right) \mathcal{A}^{1} \text { meas }_{n} \Omega^{0} .
\end{aligned}
$$

Now the claim becomes obvious.

Remark 4.4. The limit passage as $\mathcal{A}^{0} \rightarrow 0 \in \mathbb{C}^{N \times N}$ turns the transmission problem (1.2), (1.3) into the exterior Neumann boundary-value problem (1.5) for the domain $\Omega^{0}$, and the polarization matrix is always negative definite by Proposition 4.1, which remains valid after such limit passage (cf. Remark 4.2). In elasticity theory, the change of the rigidity matrix $\mathcal{A}^{0}$ that causes an unbounded growth of all its eigenvalues results in a boundary-value problem about an absolutely rigid inclusion $\Omega^{0}$, different from the Dirichlet problem (see, e.g., [30]). In the same way, the limit passage as $\mathcal{A}^{0} \rightarrow+\infty$ yields a problem with an integro-differential boundary condition

$$
\begin{aligned}
& \mathcal{L}^{1}\left(\nabla_{x}\right) u^{1}(x)=f^{1}(x), \quad x \in \Omega^{1},\left.\quad u^{1} \in \mathcal{P}\right|_{\Gamma}, \\
& \int_{\Gamma} \overline{p(x)}^{\top} \overline{\mathcal{D}}(\nu(x))^{\top} \mathcal{A}^{1} \mathcal{D}\left(\nabla_{x}\right) u^{1}(x) d x=0, \quad p \in \mathcal{P},
\end{aligned}
$$

involving the linear space (1.9) from the polynomial property (1.8). The corresponding variational problem is posed on the subspace $\left\{v \in \mathcal{H}:\left.v\right|_{\Gamma} \in \mathcal{P}\right\}$. The polarization matrix $P$ corresponding to problem (4.25) is always positive definite by Proposition 4.2 . which admits the limit passage as $\mathcal{A}^{0} \rightarrow+\infty$.

Let $\Pi$ be an orthogonal projection in $\mathbb{C}^{N}$, i.e., a matrix in $\mathbb{C}^{N \times N}$ such that $\Pi \Pi=\Pi$. Starting with the matrices $\mathcal{A}^{i}$ and $P$, we introduce the operators $\mathcal{A}_{\Pi}^{i}=\bar{\Pi}^{\top} \mathcal{A}^{i} \Pi$ and $P_{\Pi}=\bar{\Pi}^{\top} P \Pi$, acting in the subspace $\mathbb{C}_{\Pi}^{N}=\Pi \mathbb{C}^{N}$. Formulas (4.19) and (4.24) imply that, for any $b \in \mathbb{C}_{\Pi}^{N}$,

$$
\begin{aligned}
& \bar{b}^{\top} P b=-a\left(\widehat{\zeta} b, \widehat{\zeta} b ; \mathbb{R}^{n}\right)-\left(\bar{b}^{\top} \mathcal{A}^{1} b-\bar{b}^{\top} \mathcal{A}^{0} b\right) \operatorname{meas}_{n} \Omega^{0}, \\
& \bar{b}^{\top} P b=a\left(\widehat{z} b, \hat{z} b ; \mathbb{R}^{n}\right)+\left(\bar{b}^{\top} \mathcal{A}^{1} b-\bar{b}^{\top} \mathcal{A}^{1}\left(\mathcal{A}^{0}\right)^{-1} \mathcal{A}^{1} b\right) \operatorname{meas}_{n} \Omega^{0} .
\end{aligned}
$$

Therefore, our next claim becomes evident. 
Corollary 4.1. 1) If $\mathcal{A}_{\Pi}^{1} \geq \mathcal{A}_{\Pi}^{0}$, then the operator $P_{\Pi}$ is negative definite on the subspace $\mathbb{C}_{\Pi}^{n}$.

2) If $\mathcal{A}_{\Pi}^{1} \geq \bar{\Pi}^{\top} \mathcal{A}^{1}\left(\mathcal{A}^{0}\right)^{-1} \mathcal{A}^{1} \Pi$, then the operator $P_{\Pi}$ is positive definite on the subspace $\mathbb{C}_{\Pi}^{n}$.

\section{§5. The Eshelby theorem}

Since the surface $\Gamma$ is smooth, the results of [23, 31] show that the matrix-valued function $\widehat{\zeta}$, as defined in (4.17), turns out to be infinitely differentiable on the sets $\Omega^{i}$ up to the boundary $\Gamma$; here $i=0,1$. By identity (4.4) and the definition of the polynomials $q^{1}, \ldots, q^{N}$ (see formulas (4.5), (4.6)), the solution (4.18) of problem (1.2), (1.3) with the right-hand sides (4.9) $, j=1, \ldots, N$, can be written as

$$
\widehat{\zeta}(x)=\eta(x)\left(\mathcal{A}^{1}-\mathcal{A}^{0}\right),
$$

where $\eta$ is a matrix solution of the same problem with $f=0$ and $g(x)=\overline{\mathcal{D}(\nu(x))}^{\top}$. The latter problem can be rephrased as follows:

$$
\begin{aligned}
& {\overline{\mathcal{D}\left(-\nabla_{x}\right)}}^{\top} \mathcal{A}^{1} \mathcal{D}\left(\nabla_{x}\right) \eta^{1}(x)=0, \quad x \in \Omega^{1}, \\
& {\overline{\mathcal{D}\left(-\nabla_{x}\right)}}^{\top} \mathcal{A}^{1} \mathcal{D}\left(\nabla_{x}\right) \eta^{1}(x) \\
& \quad=F(x):={\overline{\mathcal{D}\left(-\nabla_{x}\right)}}^{\top}\left(\mathcal{A}^{1}-\mathcal{A}^{0}\right) \mathcal{D}\left(\nabla_{x}\right) \eta^{0}(x), \quad x \in \Omega^{0}, \\
& \overline{\mathcal{D}(\nu(x))}^{\top} \mathcal{A}^{1}\left(\mathcal{D}\left(\nabla_{x}\right) \eta^{0}(x)-\mathcal{D}\left(\nabla_{x}\right) \eta^{1}(x)\right) \\
& \quad=G(x):=\overline{\mathcal{D}(\nu(x))}^{\top}\left(\mathbb{I}_{N}+\left(\mathcal{A}^{1}-\mathcal{A}^{0}\right) \mathcal{D}\left(\nabla_{x}\right) \eta^{0}(x)\right), \quad \eta^{0}(x)=\eta^{1}(x), \quad x \in \Gamma .
\end{aligned}
$$

In other words, $\eta$ solves a system of differential equations with constant coefficients in the entire space $\mathbb{R}^{n}$; the right-hand side of this system is representable as the sum of a matrix-valued function $F$ of class $L_{2}\left(\mathbb{R}^{n}\right)^{k \times N}$ with compact support and the Dirac function distributed along the surface $\Gamma$ with density $G$ (see, e.g., [23]).

We deduce an integral representation for $\eta^{0}$. For this, we formulate some known properties of the fundamental matrix (see, e.g., [27]); we present some comments on their proofs for the reader's convenience.

Lemma 5.1. We have

$$
\Phi(x)=\Phi(-x), \quad \Phi(x)=\overline{\Phi(-x)}^{\top}, \quad x \in \mathbb{R}^{n} \backslash\{0\} .
$$

Proof. The first formula in (5.3) follows from the next observation: the change $x \mapsto-x$ affects neither the left-hand nor the right-hand side of equation (3.20), in accordance with the definitions of the operator $\mathcal{L}^{1}$ and the Dirac function $\delta$. The second formula is obtained by substituting the matrices $\Phi(y), \Phi(y-x)$ in the Green formula for the domain

$$
\left\{y \in \mathbb{R}^{n}: \min \{|y-x|,|y|\}>\mathbf{r}>0\right\}
$$

(the space without two balls of small radius $\mathbf{r}$ that are centered at $y=x$ and $y=0$ ), and by the limit passage as $\mathbf{r} \rightarrow+0$, with the use of formula (3.22).

By the definition of the fundamental matrix $\Phi$, we have

$$
\begin{aligned}
\eta(x) & =\int_{\Omega^{0}} \Phi(y-x) F(y) d y+\int_{\Gamma} \Phi(y-x) G(y) d s_{y} \\
& :=\mathcal{J}\left(\Omega^{0}\right)=\int_{\Omega^{0}}\left(\overline{\mathcal{D}\left(\nabla_{y}\right)} \Phi(y-x)^{\top}\right)^{\top}\left(\mathbb{I}_{N}+\left(\mathcal{A}^{1}-\mathcal{A}^{0}\right) \mathcal{D}\left(\nabla_{y}\right) \eta^{0}(y)\right) d y .
\end{aligned}
$$

Formula (3.21) shows that the derivatives of the fundamental matrix entries behave as $O\left(|x|^{1-n}\right)$, so that the last integral in (5.4) converges absolutely for all $x \in \mathbb{R}^{n}$. 
To calculate the matrix-valued function $\tau^{0}=\mathcal{D}\left(\nabla_{x}\right) \eta^{0}$, we consider the neighborhood $\omega_{h}(x)=\left\{y: h^{-1}(y-x) \in \omega\right\}$ of a point $x \in \Omega^{0}$ in the integration set; here $\omega$ is a domain bounded by a closed Lipschitzian surface $\partial \omega$ and containing the origin, and $h>0$ is a parameter sufficiently small to ensure that $\omega_{h}(x) \subset \Omega^{0}$. As a result, we find

$$
\begin{gathered}
\tau^{0}(x)=\mathcal{D}\left(\nabla_{x}\right) \mathcal{J}\left(\omega_{h}(x)\right)+\mathcal{D}\left(\nabla_{x}\right) \mathcal{J}\left(\Omega^{0} \backslash \omega_{h}(x)\right) \\
\mathcal{D}\left(\nabla_{x}\right) \mathcal{J}\left(\omega_{h}(x)\right) \\
=\mathcal{D}\left(\nabla_{x}\right) \int_{\omega_{h}(0)}\left(\overline{\mathcal{D}\left(\nabla_{z}\right)} \Phi(z)^{\top}\right)^{\top}\left(\mathbb{I}_{N}+\left(\mathcal{A}^{1}-\mathcal{A}^{0}\right) \mathcal{D}\left(\nabla_{z}\right) \eta^{0}(z+x)\right) d z \\
=\sum_{j=1}^{n} \mathcal{D}\left(e_{(j)}\right) \int_{\omega_{h}(0)}\left(\overline{\mathcal{D}\left(\nabla_{z}\right)} \Phi(z)^{\top}\right)^{\top}\left(\mathcal{A}^{1}-\mathcal{A}^{0}\right) \mathcal{D}\left(\nabla_{z}\right) \frac{\partial \eta^{0}}{\partial z_{j}}(z+x) d z \\
=O(h), \\
\mathcal{D}\left(\nabla_{x}\right) \mathcal{J}\left(\Omega^{0} \backslash \omega_{h}(x)\right) \\
=-\int_{\partial \omega_{h}(x)} \mathcal{D}\left(\nu^{h}(y-x)\right)\left(\overline{\mathcal{D}\left(\nabla_{y}\right)} \Phi(y-x)^{\top}\right)^{\top}\left(\mathbb{I}_{N}+\left(\mathcal{A}^{1}-\mathcal{A}^{0}\right) D\left(\nabla_{y}\right) \eta^{0}(y)\right) d s_{y} \\
-\sum_{j=1}^{n} \mathcal{D}\left(e^{j}\right) \int_{\Omega^{0} \backslash \omega_{h}(x)}\left(\overline{\mathcal{D}\left(\nabla_{y}\right)} \frac{\partial \Phi^{\top}}{\partial y_{j}}(y-x)\right)^{\top}\left(\mathbb{I}_{N}+\left(\mathcal{A}^{1}-\mathcal{A}^{0}\right) \mathcal{D}\left(\nabla_{x}\right) \eta^{0}(y)\right) d y
\end{gathered}
$$

where $e_{(j)}$ is the unit vector of the $x_{j}$-axis, and $\nu^{h}$ is the outward unit normal to the boundary of $\omega_{h}(0)$. Formula (5.6) is provided by the change $y \mapsto z=y-x$, together with the boundedness of the derivatives of $\eta^{0}$ in $\overline{\Omega^{0}}$ and the weak singularity (of order $O\left(|z|^{1-n}\right)$ ) of the first derivatives of the entries of the fundamental matrix $\Phi(z)$. Applying the formula

$$
\frac{\partial}{\partial x_{j}} \int_{\omega_{h}(x)} \psi(x, y) d y=\int_{\partial \omega_{h}(x)} \nu_{j}^{h}(y-x) \psi(x, y) d s_{y}+\int_{\omega_{h}(x)} \frac{\partial \psi}{\partial x_{j}}(x, y) d y,
$$

which is well known and easily verified, we can rewrite the first integral on the right-hand side in (5.7), which arose because the integration domain in $\mathcal{J}\left(\Omega^{0} \backslash \omega_{h}(x)\right)$ is variable, as follows:

$$
\mathcal{J}_{h}^{1}(x)=\Psi\left(\mathbb{I}_{N}+\left(\mathcal{A}^{1}-\mathcal{A}^{0}\right) \tau^{0}(x)\right)+O(h)
$$

here

$$
\Psi=-\int_{\partial \omega_{h}(0)} \mathcal{D}\left(\nu^{h}(z)\right)\left(\overline{\mathcal{D}\left(\nabla_{z}\right)} \Phi(z)^{\top}\right)^{\top} d s_{z} .
$$

The error term in (5.8) can be estimated with the help of the same change $y \mapsto z=$ $y-x$ as above and the Taylor formula for the smooth matrix-valued function $\eta^{0}$. The numerical matrix (5.9) of size $N \times N$ is determined by the shape of the surface $\partial \omega$ and the fundamental matrix $\Phi$, but does not depend on $h$, because $\left|\partial \omega_{h}(0)\right|=O\left(h^{n-1}\right)$ and the integrand is $O\left(|z|^{1-n}\right)=O\left(h^{1-n}\right)$ by (3.21).

The matrix $\Psi$ is Hermitian and positive definite. The latter property will be checked later on (see formula (5.21) ); the former follows from the symmetry of the matrix $\Phi(y)$ (see Lemma 5.1) and the relation

$$
\Psi=-\sum_{j, k=1}^{n} \mathcal{D}\left(e_{(j)}\right) \int_{\mathbb{R}^{n} \backslash \omega_{1}(0)} \frac{\partial}{\partial z_{j} \partial z_{k}}\left(\chi_{\omega}(z) \Phi(z)\right) d z{\overline{\mathcal{D}\left(e_{(k)}\right)}}^{\top},
$$

obtained by application of the Gauss-Ostrogradskiı formula to the surface integral (5.9) ( $\chi_{\omega}$ is any smooth cutoff function with compact support equal to 1 near the set $\overline{\omega_{1}(0)}$ ). 
The last integral $\mathcal{J}_{h}^{2}(x)$ in (5.7) will be transformed as follows:

$$
\begin{aligned}
\mathcal{J}_{h}^{2}(x)= & \sum_{j=1}^{n} \mathcal{D}\left(e_{(j)}\right) \int_{\Omega^{0} \backslash \omega_{h}(x)}\left(\overline{\mathcal{D}\left(\nabla_{y}\right)} \frac{\partial \Phi^{\top}}{\partial y_{j}}(y-x)\right)^{\top} \\
& \times\left(\mathcal{A}^{1}-\mathcal{A}^{0}\right)\left(\tau^{0}(x)-\tau^{0}(y)\right) d y \\
- & \sum_{j=1}^{n} \mathcal{D}\left(e_{(j)}\right) \int_{\Omega^{0} \backslash \omega_{h}(x)}\left(\overline{\mathcal{D}\left(\nabla_{y}\right)} \frac{\partial \Phi^{\top}}{\partial y_{j}}(y-x)\right)^{\top} d y \\
& \times\left(\mathbb{I}_{6}+\left(\mathcal{A}^{1}-\mathcal{A}^{0}\right) \mathcal{D}\left(\nabla_{x}\right) \eta^{0}(x)\right) \\
= & : \mathcal{J}_{h}^{21}(x)-\mathcal{J}_{h}^{22}(x) .
\end{aligned}
$$

Since the matrix-valued function $\tau^{0}$ is smooth in $\overline{\Omega^{0}}$ and $\left|\tau^{0}(x)-\tau^{0}(y)\right| \leq c|x-y|$, the integral $\mathcal{J}_{0}^{21}(x)$ over the domain $\Omega^{0}$ converges absolutely, and the first term in the middle part of (5.10) admits a limit passage as $h \rightarrow+0$, which leads to the difference integral operator

$$
\mathcal{J}_{0}^{21}(x)=\int_{\Omega^{0}} \mathcal{K}(y-x)\left(\tau^{0}(x)-\tau^{0}(y)\right) d y,
$$

where the kernel $\mathcal{K}(y-x)$ has a singularity of order $O\left(|y-x|^{-n}\right)$. If the function $\tau^{0}$ is smooth, then the integral (5.11) converges absolutely.

The coefficient

$$
\mathcal{J}^{0}\left(\Omega^{0} \backslash \omega_{h}(x)\right):=\sum_{j=1}^{n} \mathcal{D}\left(e_{(j)}\right) \int_{\Omega^{0} \backslash \omega_{h}(x)}\left(\overline{\mathcal{D}\left(\nabla_{y}\right)} \frac{\partial \Phi^{\top}}{\partial y_{j}}(y-x)\right)^{\top} d y
$$

of the matrix $\mathbb{I}_{N}+\left(\mathcal{A}^{1}-\mathcal{A}^{0}\right) \mathcal{D}\left(\nabla_{x}\right) \eta^{0}(x)=\mathbb{I}_{N}+\left(\mathcal{A}^{1}-\mathcal{A}^{0}\right) \tau^{0}(x)$ in the expression $\mathcal{J}_{h}^{22}(x)$ does not depend on $h$. Indeed, for $H>h>0$ we have

$$
\begin{aligned}
\mathcal{J}^{0}\left(\omega_{H}(x) \backslash \omega_{h}(x)\right)= & \int_{\omega_{H}(x) \backslash \omega_{h}(x)}\left(\overline{\mathcal{D}\left(\nabla_{y}\right)} \frac{\partial \Phi^{\top}}{\partial y_{j}}(y-x)\right)^{\top} d y \\
= & \int_{\partial \omega_{H}(x)}\left(\overline{\mathcal{D}\left(\nu^{H}(y-x)\right)} \frac{\partial \Phi^{\top}}{\partial y_{j}}(y-x)\right)^{\top} d s_{y} \\
& -\int_{\partial \omega_{h}(x)}\left(\overline{\mathcal{D}\left(\nu^{h}(y-x)\right)} \frac{\partial \Phi^{\top}}{\partial y_{j}}(y-x)\right)^{\top} d s_{y} \\
= & 0 .
\end{aligned}
$$

The coincidence of the integrals over the surfaces $\partial \omega_{H}(x)$ and $\partial \omega_{h}(x)$ is ensured by the homogeneity of order $1-n$ of their (common) integrand. Thus, the integral (5.12) gives us some $(N \times N)$-matrix-valued function in the domain $\mathbf{K}$. Passing to the limit in (5.5) and using the transformations made above, we arrive at the integral equation

$$
\tau^{0}(x)=\Psi\left(\mathbb{I}_{N}+\left(\mathcal{A}^{1}-\mathcal{A}^{0}\right) \tau^{0}(x)\right)+\mathbf{K}(x) \tau^{0}(x)+\int_{\Omega^{0}} \mathcal{K}(y-x)\left(\tau^{0}(x)-\tau^{0}(y)\right) d y
$$

If we manage to find a vector-valued function $\tau^{0}$ smooth in $\overline{\Omega^{0}}$ and satisfying (5.14), then we shall be able to use formula (5.4) (without its middle part) to recover the solution $\eta$ of problem (5.2), and the classical results about integral operators (see, e.g., [32]) show that $\eta$ belongs to the space $H_{\text {loc }}^{1}\left(\mathbb{R}^{n}\right)^{k}$.

Since $\left|\nabla_{x} \Phi(x)\right|=O\left(|x|^{1-n}\right)$ and integration in (5.4) is over a bounded domain, the restriction of $\eta^{1}$ to $\Omega^{1}$ admits the estimate

$$
\left|\eta^{1}(x)\right| \leq c|x|^{1-n}, \quad x \in \mathbb{R}^{n} \backslash \mathbb{B}_{R}^{n}
$$




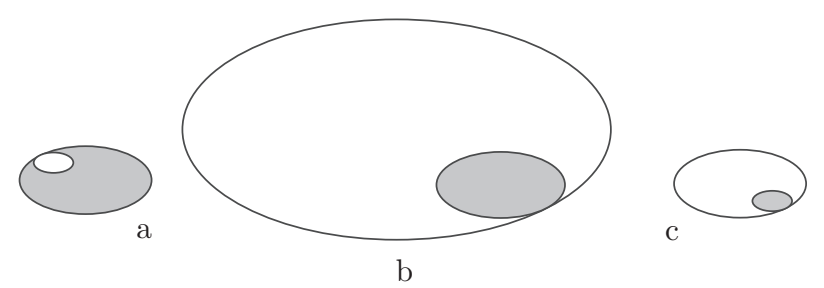

FigurE 2. Transformation of ellipsoids.

Now, applying general results about the lifting of differential properties and the behavior at infinity of solutions of boundary-value problems and transmission problems (see [33, 24, 29 and [34, 23, 31], respectively), we see that $\eta$ belongs to the weighted class $\mathcal{V}_{\beta}^{l+1}\left(\Omega^{0} \cup\right.$ $\left.\Omega^{1}\right)^{k}$. Since, by Proposition 3.2 , the uniqueness theorem is valid for this class, the recovered function $\eta$ coincides with the true solution of problem (5.2).

In general, to find a solution of the integral equation (5.14) is as difficult as to solve the differential problem (5.2) itself. Therefore, the following observation, made in [14] for elasticity theory problems, looks unexpected: in the case of similar ellipses $\Omega^{0}$ and $\omega_{h}$, the equation admits a constant solution $\tau^{0}$, and the matrix $\mathbf{K}$ turns out to be zero in this case (see also [15, 16]).

Let $\Omega^{0}$ be the ellipsoid (2.6). We verify that expression (5.12) equals zero for all $x \in \Omega^{0}$. For this, we fix a point $x^{0} \in \Omega^{0}$ and choose the sizes $h>0$ and $H>0$ so that the ellipsoids $\omega_{h}\left(x^{0}\right)$ and $\omega_{H}\left(x^{0}\right)$ touch the ellipsoid $\Omega^{0}$, similar to them, from inside and from outside, respectively (see Figure 2, a, b). Simple geometric argument 2 show that the tangent points $x^{h}$ and $x^{H}$ of the pairs of surfaces $\partial \omega_{h}\left(x^{0}\right), \partial \Omega^{0}$ and $\partial \omega_{H}\left(x^{0}\right), \partial \Omega^{0}$ lie on the line passing through the centers $x^{0}$ and $x=0$ of the ellipsoids $\omega_{h}\left(x^{0}\right), \omega_{H}\left(x^{0}\right)$ and $\Omega^{0}$, respectively. Applying formula (5.13), i.e., adding a zero integral, and using the fact that $\left(\omega_{H}(x) \backslash \omega_{h}(x)\right) \backslash\left(\Omega^{0} \backslash \omega_{h}(x)\right)=\omega_{H}(x) \backslash \Omega^{0}$, we obtain

$$
\begin{aligned}
\mathcal{J}^{0}\left(\Omega^{0} \backslash \omega_{h}(x)\right) & =\frac{1}{2} \mathcal{J}^{0}\left(\Omega^{0} \backslash \omega_{h}(x)\right)-\frac{1}{2}\left(\mathcal{J}^{0}\left(\omega_{H}(x) \backslash \omega_{h}(x)\right)-\mathcal{J}^{0}\left(\Omega^{0} \backslash \omega_{h}(x)\right)\right) \\
& =\frac{1}{2} \mathcal{J}^{0}\left(\Omega^{0} \backslash \omega_{h}(x)\right)-\frac{1}{2} \mathcal{J}^{0}\left(\omega_{H}(x) \backslash \Omega^{0}\right) .
\end{aligned}
$$

Observe that, for the same reason as before, the contraction and shift of the Cartesian coordinate system that take $\omega_{H}(x)$ and $\Omega^{0}$ to $\Omega^{0}$ and $\left\{z:-z \in \omega_{h}(x)\right\}$, respectively (see Figure 2, b, c), do not affect the last integral in (5.13). Therefore, the integral on the left in (5.15) becomes equal to the half-difference of the integrals over the sets depicted in Figure 2, a, c, and this quantity vanishes because the fundamental matrix is even (see the first formula in (5.3) $)$. Thus, indeed,

$$
\mathbf{K}(x)=0 \in \mathbb{C}^{N \times N}, \quad x \in \Omega^{0} .
$$

Now, it is not difficult to find a constant solution

$$
\tau^{0}=\left(\mathbb{I}_{N}-\Psi\left(\mathcal{A}^{1}-\mathcal{A}^{0}\right)\right)^{-1} \Psi
$$

that belongs to the kernel of the integral operator (5.11), provided that the matrix

$$
\mathbb{I}_{N}-\Psi \mathcal{A}^{1}+\Psi \mathcal{A}^{0} \in \mathbb{C}^{N \times N}
$$

is nonsingular. The first two terms do not depend on the matrix $\mathcal{A}^{0}$ in the inclusion $\Omega^{0}$; therefore, the matrix (5.17) is invertible at least for a.e. $\mathcal{A}^{0}$. For instance, fixing

\footnotetext{
${ }^{2}$ The simplest way of verification consists in making an affine transformation $x \mapsto\left(\ell_{1}^{-1} x_{1}, \ldots, \ell_{n}^{-1} x_{n}\right)$ and passing to balls, for which the required geometric facts are obvious.
} 
an $(N \times N)$-matrix $A^{0}$, Hermitian and positive definite, we see that if $\mathcal{A}^{0}=\mu A^{0}$ and $\mu>0$ is large, then the matrix (5.17) is invertible. First, we shall deal precisely with such matrices, and then the result will be extended to all coefficient matrices $\mathcal{A}^{0}$.

We define the transfer matrix

$$
T=\tau^{0}=\mathcal{D}\left(\nabla_{x}\right) \eta^{0}(x) \in \mathbb{C}^{N \times N} .
$$

The name is justified by formula (2.7), which is implied by the relations

$$
\begin{aligned}
\zeta(x) \varepsilon^{\infty} & =\left(\overline{\mathcal{D}(x)}^{\top}+\widehat{\zeta}(x)\right) \varepsilon^{\infty}=\left(\overline{\mathcal{D}(x)}^{\top}+\eta(x)\left(\mathcal{A}^{1}-\mathcal{A}^{0}\right)\right) \varepsilon^{\infty} \\
\varepsilon^{0} & =\mathcal{D}\left(\nabla_{x}\right) \zeta^{0}(x) \varepsilon^{\infty}=\left(\mathbb{I}_{N}+\mathcal{D}\left(\nabla_{x}\right) \eta^{0}(x)\left(\mathcal{A}^{1}-\mathcal{A}^{0}\right)\right) \varepsilon^{\infty},
\end{aligned}
$$

valid due to (5.1) and (4.4). Using the Green formula (3.1) and the transmission condition (1.3) with the right-hand side $\overline{\mathcal{D}(\nu(x))}^{\top}$ for $\eta$, we obtain

$$
\begin{aligned}
\left(\mathcal{A}^{1} \mathcal{D}\right. & \left.\left(\nabla_{x}\right) \eta^{1}, \mathcal{D}\left(\nabla_{x}\right) \eta^{1}\right)_{\Omega^{1}}+\left(\mathcal{A}^{0} \mathcal{D}\left(\nabla_{x}\right) \eta^{0}, \mathcal{D}\left(\nabla_{x}\right) \eta^{0}\right)_{\Omega^{0}} \\
& =\left(\mathcal{N}^{0} \eta^{0}-\mathcal{N}^{1} \eta^{1}, \eta\right)_{\Gamma} \\
& =\int_{\Gamma} \overline{\eta(x)}^{\top} \overline{\mathcal{D}(x)}^{\top} d s_{x}={\overline{\int_{\Gamma} \mathcal{D}(x) \eta^{0}(x) d s_{x}}}^{\top}={\overline{\int_{\Omega^{0}} \mathcal{D}\left(\nabla_{x}\right) \eta^{0}(x) d x}}^{\top} \\
& =\left(\tau^{0}\right)^{\top} \operatorname{meas}_{n} \Omega^{0}=T^{\top} \operatorname{meas}_{n} \Omega^{0} .
\end{aligned}
$$

On the left in (5.19) we have the Gram matrix built by the (linearly independent) columns of the matrix $\eta=\left(\eta^{(1)}, \ldots, \eta^{(N)}\right)$ with the help of the scalar product (2.5). It follows that the link matrix $T$ is Hermitian and positive definite. Thus, the last factor $\Psi$ in (5.16) is a nonsingular matrix, so that

$$
\mathbb{O}_{N}<\Psi^{-1}\left(\mathbb{I}_{N}-\Psi\left(\mathcal{A}^{1}-\mathcal{A}^{0}\right)\right)=\Psi^{-1}-\mathcal{A}^{1}+\mathcal{A}^{0} .
$$

Here $\mathbb{O}_{N}$ is the null $(N \times N)$-matrix, and the first inequality expresses the fact that the matrix on the right in (5.20) is positive definite. As has already been mentioned, the deduction of (5.20) is valid if the matrix (5.17) is nonsingular. Suppose that $\mathcal{A}^{0}=\mu A^{0}$, as before. The algebraic equation

$$
\operatorname{det}\left(\mathbb{I}_{N}-\Psi \mathcal{A}^{0}+\mu \Psi A^{0}\right)=0
$$

has $N$ roots $\mu \in \mathbb{C}$. Therefore, there exists a real number $\mu\left(A^{0}\right)>0$ such that the matrix (5.17) is invertible for $\mu \in\left(0, \mu\left(A^{0}\right)\right)$, whence

$$
\Psi^{-1}-\mathcal{A}^{1}+\mu A^{0}>\mathbb{O}_{N}
$$

Letting $\mu$ tend to zero, we arrive at the relations

$$
\Psi^{-1}-\mathcal{A}^{1} \geq \mathbb{O}_{N} \quad \Rightarrow \quad \Psi>\mathbb{O}_{N},
$$

which entail the next statement.

Lemma 5.2. Formula (5.20) and the invertibility of the matrix (5.17) occur for all coefficient matrices $\mathcal{A}^{1}$ and $\mathcal{A}^{0}$.

We denote by $\Psi$ the positive square root of the positive definite matrix $\Psi^{1 / 2}$; using (5.16) and (5.18), we can write the link matrix in a symmetric form:

$$
\begin{aligned}
T & =\left(\mathbb{I}_{N}-\Psi\left(\mathcal{A}^{1}-\mathcal{A}^{0}\right)\right)^{-1} \Psi^{1 / 2} \Psi^{1 / 2} \\
& =\left(\Psi^{-1 / 2}-\Psi^{-1 / 2} \Psi\left(\mathcal{A}^{1}-\mathcal{A}^{0}\right) \Psi^{1 / 2} \Psi^{-1 / 2}\right)^{-1} \Psi^{1 / 2} \\
& =\Psi^{1 / 2}\left\{\left(\mathbb{I}_{N}-\Psi^{1 / 2}\left(\mathcal{A}^{1}-\mathcal{A}^{0}\right)\right) \Psi^{1 / 2}\right\}^{-1} \Psi^{1 / 2} .
\end{aligned}
$$

Note that, by the inequalities (5.21) and $\mathcal{A}^{0}>\mathbb{O}_{N}$, the matrix in braces is positive definite. 
Lemma 5.2 ensures the existence of a constant solution (5.16) for all $\mathcal{A}^{1}$ and $\mathcal{A}^{0}$. Thus, we have proved the next statement, which generalizes the Eshelby theorem, known in elasticity theory.

Theorem 5.1. Let $\Omega^{0}$ be the ellipsoid (2.6), and let $\zeta\left(\varepsilon^{\infty} ; \cdot\right)$ be a solution of the homogeneous function problem (1.2), (1.3) with the following conditions at infinity:

$$
\zeta\left(\varepsilon^{\infty} ; x\right)=\overline{\mathcal{D}(x)}^{\top}+\widehat{\zeta}\left(\varepsilon^{\infty} ; x\right), \quad \widehat{\zeta}\left(\varepsilon^{\infty} ; \cdot\right) \in \mathcal{V}_{\beta}^{l+1}\left(\Omega^{0} \cup \Omega^{1}\right)^{k}
$$

where $l \in \mathbb{N}$, and the weight index $\beta$ is as in (3.18); i.e., the remainder $\widehat{\zeta}\left(\varepsilon^{\infty} ; x\right)$ decays as $|x| \rightarrow \infty$. Then the matrix $\mathcal{D}\left(\nabla_{x}\right) \zeta^{0}\left(\varepsilon^{\infty} ; x\right)$ is constant inside the ellipsoid, and we have

$$
\mathcal{D}\left(\nabla_{x}\right) \zeta^{0}\left(\varepsilon^{\infty} ; x\right)=\left(\mathbb{I}_{N}+T\left(\mathcal{A}^{1}-\mathcal{A}^{0}\right)\right) \varepsilon^{\infty}, \quad x \in \Omega^{0},
$$

where $T$ is the Hermitian and positive definite link matrix of size $N \times N$ defined by (5.18) and (5.16), or by (5.22).

By relations (5.1) and (4.18), the polarization $P$ can be written as $Q\left(\mathcal{A}^{1}-\mathcal{A}^{0}\right)$, where $Q$ is the matrix in the expansion

$$
\eta^{1}(x)=\left(\overline{\mathcal{D}\left(\nabla_{x}\right)} \Phi(x)^{\top}\right)^{\top} Q+\widetilde{\eta}^{1}(x), \quad \widetilde{\eta}^{1} \in V_{\gamma}^{l+1}\left(\Omega^{1}\right)^{k \times N}
$$

of the solution $\eta$ of problem (5.2) (cf. (4.18)). It is not hard to compute $Q$ by the method of [28, because $\overline{\mathcal{D}}(x)^{\top}$ is a solution of the homogeneous $(F=0$ and $G=0$ ) system of differential equations (5.2). Repeating the calculations from the proof of Proposition 4.1 (with significant simplifications), we find that

$$
\begin{aligned}
-Q & =\int_{\Omega^{0}} \mathcal{D}(x) F(x) d x+\int_{\Gamma} \mathcal{D}(x) G(x) d s_{x} \\
& =\int_{\Omega^{0}}\left(\overline{\mathcal{D}\left(\nabla_{x}\right)} \mathcal{D}(x)^{\top}\right)^{\top}\left(\mathcal{A}^{1}-\mathcal{A}^{0}\right) \mathcal{D}\left(\nabla_{x}\right) \eta^{0}(x) d x+\int_{\Gamma} \mathcal{D}(x) \overline{\mathcal{D}(\nu(x))} \\
& =\int_{\Omega^{0}}\left(\mathcal{A}^{1}-\mathcal{A}_{x}\right) \tau^{0}(x) d x+\int_{\Omega^{0}} \overline{\mathcal{D}\left(\nabla_{x}\right)} \mathcal{D}(x)^{\top} d x \\
& =\operatorname{meas}_{n} \Omega^{0}\left(\left(\mathcal{A}^{1}-\mathcal{A}^{0}\right) T+\mathbb{I}_{N}\right) .
\end{aligned}
$$

Consequently,

$$
P=-\operatorname{meas}_{n} \Omega^{0}\left(\mathcal{A}^{1}-\mathcal{A}^{0}+\left(\mathcal{A}^{1}-\mathcal{A}^{0}\right) T\left(\mathcal{A}^{1}-\mathcal{A}^{0}\right)\right)
$$

Plugging the expression (5.22) for the link matrix $T$ into (5.23), we obtain the formula

$$
\begin{aligned}
P= & -\operatorname{meas}_{n} \Omega^{0} \\
& \times\left(\mathcal{A}^{1}-\mathcal{A}^{0}+\left(\mathcal{A}^{1}-\mathcal{A}^{0}\right) \Psi^{1 / 2}\left(\mathbb{I}_{N}-\Psi^{1 / 2}\left(\mathcal{A}^{1}-\mathcal{A}^{0}\right) \Psi^{1 / 2}\right)^{-1} \Psi^{1 / 2}\left(\mathcal{A}^{1}-\mathcal{A}^{0}\right)\right) \\
= & -\operatorname{meas}_{n} \Omega^{0}\left(\mathcal{A}^{1}-\mathcal{A}^{0}+\left(\mathcal{A}^{1}-\mathcal{A}^{0}\right)\left(\Psi^{-1}-\mathcal{A}^{1}+\mathcal{A}^{0}\right)^{-1}\left(\mathcal{A}^{1}-\mathcal{A}^{0}\right)\right) .
\end{aligned}
$$

If $\mathcal{A}^{0}=0$ and $\Omega^{0}$ is a cavity, then the polarization matrix for the exterior Neumann problem (1.5) takes the simple form

$$
\begin{aligned}
P & =-\operatorname{meas}_{n} \Omega^{0}\left(\mathcal{A}^{1}+\mathcal{A}^{1} \Psi^{1 / 2}\left(\mathbb{I}_{N}-\Psi^{1 / 2} \mathcal{A}^{1} \Psi^{1 / 2}\right)^{-1} \Psi^{1 / 2} \mathcal{A}^{1}\right) \\
& =-\operatorname{meas}_{n} \Omega^{0}\left(\mathcal{A}^{1}+\mathcal{A}^{1}\left(\Psi^{-1}-\mathcal{A}^{1}\right)^{-1} \mathcal{A}^{1}\right) .
\end{aligned}
$$

Note that all matrices whose inverses occur in (5.24) and (5.25) are indeed nonsingular by what has been proved above. 


\section{§6. PATCh OPTIMiZATiOn}

Consider the Dirichlet problem (2.1) -(2.3) with the transmission conditions (2.2) on the boundary $\partial \omega_{h}$ of a small inclusion $\omega_{h}$. Suppose that $f \in C^{0, \alpha}(\Xi)^{k}$; then, by [33], a unique bounded solution of the limiting $(h=0)$ problem

$$
\mathcal{L}\left(\nabla_{x}\right) v_{(0)}(x)=f(x), \quad x \in \Xi, \quad v_{(0)}(x)=0, \quad x \in \partial \Xi,
$$

belongs to the Hölder class $C^{2, \alpha}(\Xi)^{k}$, which has the norm

$$
\left\|v ; C^{l, \alpha}(\Xi)\right\|=\sum_{j=0}^{l} \sup _{x \in \Xi}\left|\nabla_{x}^{j} v(x)\right|+\sup _{x, y \in \Xi}|x-y|^{-\alpha}\left|\nabla_{x}^{l} v(x)-\nabla_{y}^{l} v(y)\right|, \quad l \in \mathbb{N}_{0}, \quad \alpha \in(0,1) .
$$

There are two methods for constructing the asymptotics of solutions of elliptic, singularly perturbed boundary-value problems, namely, involving matched asymptotic expansions and compound asymptotic expansions (see, e.g., the books 35 and 36, respectively). For the most part, these methods are equivalent. In the required generality, the problem about a small cavity or inclusion was studied with the help of one or the other method in [37, 38, 9, 39], and simple examples were considered in [37, 40, 8, 41] and other publications. Therefore, the result of the asymptotic analysis of the energy functional (2.8) will be formulated below without accompanying calculations and the justification procedure, but with only brief explanations in Remarks 6.1 and 6.2 .

Proposition 6.1. The functional (2.8) evaluated at the solution $v_{(h)} \in \stackrel{\circ}{H}^{1}(\Xi)^{k}$ of problem (2.1)-(2.3) admits the asymptotic expansion

$$
\begin{aligned}
& E\left(v_{(h)}, f ; \Xi(h) \cup \omega_{h}\right) \\
& \quad=E\left(v_{(0)}, f ; \Xi\right)-\frac{1}{2} h^{n}{\overline{\mathcal{D}\left(\nabla_{x}\right) v_{(0)}(0)}}^{\top} P \mathcal{D}\left(\nabla_{x}\right) v_{(0)}(0)+\widetilde{E}(h),
\end{aligned}
$$

where $v_{(0)} \in C^{2, \alpha}(\Xi)^{k}$ is the solution of the limiting problem (6.1) in the domain $\Xi$ and $P$ is the polarization matrix of the inclusion $\Omega^{0}=\omega_{1}$ in the ambient space $\Omega^{1}=\mathbb{R}^{n} \backslash \overline{\omega_{1}}$ (see Propositions 4.1, 4.2 and formula (4.18), and the remainder in (6.2) satisfies the estimate

$$
|\widetilde{E}(h)| \leq c h^{n+\alpha}\left\|f ; C^{0, \alpha}(\Xi)\right\|^{2},
$$

where $c$ is a constant independent of the vector-valued function $f$ and the parameter $h \in\left(0, h_{0}\right], h_{0}>0$.

Remark 6.1. In accordance with the notation (4.5), the Taylor formula for $v_{(0)}$ can be written as follows:

$$
v_{(0)}(x)=\sum_{p=1}^{k(n+1)} c_{(0)}^{j} q^{j}(x)+Q(x)+O\left(|x|^{3}\right) ;
$$

here $Q(t x)=t^{2} Q(x)$ is a quadratic vector-valued function and $\mathcal{L}^{1}\left(\nabla_{x}\right) Q(x)=f(0)$. By (4.4), the column of coefficients $c_{(0)}^{\mathcal{D}}=\left(c_{(0)}^{1}, \ldots, c_{(0)}^{N}\right)^{\top}$ is calculated by the formula

$$
c_{(0)}^{\mathcal{D}}=\mathcal{D}\left(\nabla_{x}\right) v_{(0)}(0) \text {. }
$$

Since the terms $c_{(0)}^{N+1} q^{N+1}, \ldots, c_{(0)}^{k n+1} q^{k n+1}$ in the sum (6.4) are killed by the operator $\mathcal{D}\left(\nabla_{x}\right)$, the main discrepancy of the vector-valued function $v_{(0)}$ in the singularly perturbed problem (2.1) -(2.3) arises in the transmission conditions (2.2) and, in the leading approximation, turns out to be equal to $\overline{\mathcal{D}(\nu(x))}^{\top}\left(\mathcal{A}^{1}-\mathcal{A}^{0}\right) c_{(0)}^{\mathcal{D}}$. This expression can be compensated by the solution

$$
h Z^{1}\left(h^{-1} x\right)=h \widehat{\zeta}\left(h^{-1} x\right) c_{(0)}^{\mathcal{D}}=h \widehat{\zeta}\left(h^{-1} x\right) \mathcal{D}\left(\nabla_{x}\right) v_{(0)}(0)
$$


of the boundary layer type. If $\mathcal{A}^{0}>\mathbb{O}_{N}$, then problem (1.2), (1.3) for the next term $h^{2} Z^{2}\left(h^{-1} x\right)$ of the boundary layer acquires the following right-hand sides:

$$
\begin{gathered}
f^{1}(\xi)=0, \quad f^{0}(\xi)=f(0)-\mathcal{L}^{0}\left(\nabla_{\xi}\right) Q(\xi)={\overline{\mathcal{D}\left(-\nabla_{\xi}\right)}}^{\top}\left(\mathcal{A}^{1}-\mathcal{A}^{0}\right) \mathcal{D}\left(\nabla_{\xi}\right) Q(\xi), \\
g(\xi)=\overline{\mathcal{D}(\nu(\xi))}{ }^{\top}\left(\mathcal{A}^{1}-\mathcal{A}^{0}\right) \mathcal{D}\left(\nabla_{\xi}\right) Q(\xi) .
\end{gathered}
$$

Since

$$
\int_{\Omega^{0}}{\overline{\mathcal{D}}\left(-\nabla_{\xi}\right)}^{\top}\left(\mathcal{A}^{1}-\mathcal{A}^{0}\right) \mathcal{D}\left(\nabla_{\xi}\right) Q(\xi) d x+\int_{\Gamma} \overline{\mathcal{D}(\nu(\xi))}^{\top}\left(\mathcal{A}^{1}-\mathcal{A}^{0}\right) \mathcal{D}\left(\nabla_{\xi}\right) Q(\xi) d s_{x}=0
$$

the method of 28] (cf. the calculations (4.13) in the proof of Proposition 4.1) shows that the coefficients $c_{0,1}, \ldots, c_{0, k}$ in the expansion (3.24) for $Z^{2}$ vanish, and hence, the second term $h^{2} Z^{2}\left(h^{-1} x\right)$ of the boundary layer decays sufficiently fast at infinity, with the rate $O\left(|\xi|^{1-n}\right)=O\left(h^{2} h^{n-1}|x|^{1-n}\right)$, and its discrepancy in the Dirichlet boundary condition (2.3) is of order $h^{n+1}$, which is larger than the order $h^{n}$ of the leading part of the discrepancy of the first term $h Z^{1}\left(h^{-1} x\right)$ (cf. Remark 4.3). As a result, in accordance with formulas (4.18) and (6.5), for $Z^{1}$ the asymptotic correction term $h^{n} v^{(n)}(x)$ of smooth type is found from the boundary-value problem

$$
\begin{gathered}
\mathcal{L}^{1}\left(\nabla_{x}\right) v^{(n)}(x)=0, \quad x \in \Xi, \\
v^{(n)}(x)=-\left(\overline{\mathcal{D}\left(\nabla_{x}\right)} \Phi(x)^{\top}\right)^{\top} P \mathcal{D}\left(\nabla_{x}\right) v_{(0)}(0), \quad x \in \partial \Xi .
\end{gathered}
$$

The asymptotics of the functional (2.8) can be calculated as follows. First, by application of the Green formula, the solution $v_{(h)}$ is replaced with its asymptotic representation

$$
\begin{aligned}
& E\left(v_{(h)}, f ; \Xi(h) \cup \omega_{h}\right)=-\frac{1}{2} \operatorname{Re}\left(f, v_{(h)}\right) \Xi \\
& \quad=-\frac{1}{2} \operatorname{Re}\left(f, v_{(0)}+h \widehat{\zeta}\left(h^{-1} x\right) \mathcal{D}\left(\nabla_{x}\right) v_{(0)}(0)+h^{n} v^{(n)}(x)\right)_{\Xi}+\cdots
\end{aligned}
$$

Note that, by the same Green formula, the expression on the right in (6.9) is equal to $\frac{1}{2}\left(\mathcal{A D}(\nabla) v_{(h)}, \mathcal{D}(\nabla) v_{(h)}\right)_{\Xi}$ and is real because the form is Hermitian; the dots hide infinitesimally small quantities of order $O\left(h^{n+\alpha}\right)$ - this estimate is ensured by the results of the paper cited above. Next we use the relations

$$
\begin{aligned}
& -\frac{1}{2} \operatorname{Re}\left(f, v_{(0)}\right)_{\Xi}=E\left(v_{(0)}, f ; \Xi\right), \\
& h\left|\left(f, \widehat{\zeta}\left(h^{-1} x\right) \mathcal{D}\left(\nabla_{x}\right) v_{(0)}(0)\right)_{\Xi}\right| \leq \operatorname{ch}\left|\mathcal{D}\left(\nabla_{x}\right) v_{(0)}(0)\right| \max _{x \in \Xi}|f(x)|\left|\int_{\Xi}\left(1+h^{-1}|x|\right)^{-n} d x\right| \\
& \leq \operatorname{ch}^{n}(1+|\ln h|)\left\|f ; C^{0, \alpha}(\Xi)\right\|^{2}
\end{aligned}
$$

(see Remark 4.3). Finally, we use (4.18) and (3.21) to compute the remaining integral:

$$
\begin{aligned}
-\frac{1}{2} & \operatorname{Re}\left(f, h\left(\overline{\mathcal{D}\left(h \nabla_{x}\right)} \Phi\left(h^{-1} x\right)^{\top}\right)^{\top} P \mathcal{D}\left(\nabla_{x}\right) v_{(0)}(0)+h^{n} v^{(n)}(x)\right)_{\Xi(h)} \\
& =-\frac{1}{2} h^{n} \operatorname{Re}\left(f,\left(\overline{\mathcal{D}\left(\nabla_{x}\right)} \Phi(x)^{\top}\right)^{\top} P \mathcal{D}\left(\nabla_{x}\right) v_{(0)}(0)+v^{(n)}(x)\right)_{\Xi(h)} \\
& =-\frac{1}{2} h^{n} \operatorname{Re}\left(f, V^{(n)}(x)\right)_{\Xi(h)^{\prime}} .
\end{aligned}
$$

For this we observe that, by condition (6.8), the sum

$$
V^{(n)}(x)=\left(\overline{\mathcal{D}\left(\nabla_{x}\right)} \Phi(x)^{\top}\right)^{\top} P \mathcal{D}\left(\nabla_{x}\right) v_{(0)}(0)+v^{(n)}(x)
$$


satisfies the homogeneous Dirichlet problem (6.1) in the punctured domain $\Xi \backslash\{0\} \subset \Xi(\delta)$. Now, with the help of the Green formula in the domain $\Xi(\delta)=\Xi \backslash \overline{\omega_{\delta}}$, we obtain the relation

$$
\begin{aligned}
\operatorname{Re}\left(f, V^{(n)}\right)_{\Xi(\delta)} & =\operatorname{Re}\left(\mathcal{L}^{1} v_{(0)}, V^{(n)}\right)_{\Xi(\delta)}-\operatorname{Re}\left(v_{(0)}, \mathcal{L}^{1} V^{(n)}\right)_{\Xi(\delta)} \\
& =\operatorname{Re}\left(\mathcal{N}^{1} v_{(0)}, V^{(n)}\right)_{\partial \omega_{\delta}}-\operatorname{Re}\left(v_{(0)}, \mathcal{N}^{1} V^{(n)}\right)_{\partial \omega_{\delta}} \\
& =-\operatorname{Re}\left(v_{(0)}, \mathcal{N}^{1}\left(\overline{\mathcal{D}\left(\nabla_{x}\right)} \Phi(x)^{\top}\right)^{\top} P \mathcal{D}\left(\nabla_{x}\right) v_{(0)}(0)\right)_{\partial \omega_{\delta}}+O(\delta) \\
& =\left(\overline{\mathcal{D}\left(\nabla_{x}\right) v_{(0)}(0)}\right)^{\top} P \mathcal{D}\left(\nabla_{x}\right) v_{(0)}(0)+O(\delta) .
\end{aligned}
$$

It should be noted that the last identity is based on the calculations (4.15), (4.13) in the proof of Proposition 4.1 and $\left(\mathcal{N}^{1} v_{(0)}, V^{(n)}\right)_{\partial \omega_{\delta}}=O(\delta)$ because $v_{(0)} \in C^{2, \alpha}(\Xi)^{k}$. Also, the expression involving the (Hermitian) polarization matrix $P$ is real. Collecting the above formulas and letting $\delta \rightarrow+0$, we arrive at the asymptotic expansion (6.2), (6.3).

Remark 6.2. For the singularly perturbed mixed boundary-value problem

$$
\begin{aligned}
\mathcal{L}^{1}\left(\nabla_{x}\right) v_{(h)}(x) & =f(x), \quad x \in \Xi(h), \quad v_{(h)}(x)=0, \quad x \in \partial \Xi, \\
\mathcal{N}^{1}\left(x, \nabla_{x}\right) v_{(h)}(x) & =0, x \in \partial \omega_{h},
\end{aligned}
$$

Proposition 6.1 can be deduced via the limit passage as $\mathcal{A}^{0} \rightarrow \mathbb{O}_{N}$, but this method meets calculational difficulties, so that it is convenient to deduce the result independently. Problem (6.12) gives rise to the exterior Neumann problem (1.5) for the description of the boundary layer phenomenon, and the right-hand sides $g^{1}$ and $g^{2}$ for the components $Z^{1}$ and $Z^{2}$ take the form

$$
g^{1}(\xi)=\overline{\mathcal{D}(\nu(\xi))} \mathcal{A}^{1} \mathcal{D}\left(\nabla_{x}\right) v_{(0)}(0), \quad g^{2}(\xi)=-\mathcal{N}^{1}\left(\xi, \nabla_{\xi}\right) Q(\xi) .
$$

In other words, formula (6.5) for $Z^{1}$ remains valid, but identity (6.6) is replaced with the following:

$$
\int_{\Gamma} g^{2}(\xi) d s_{\xi}=-\int_{\Omega^{0}} \mathcal{L}^{1}\left(\nabla_{\xi}\right) Q(\xi) d \xi=-f(0) \operatorname{meas}_{n} \Omega^{0} .
$$

Consequently, the same method [28] as before shows that the second term of the boundary layer type has the following asymptotics:

$$
\begin{aligned}
Z^{2}(\xi) & =-\Phi(\xi) f(0) \operatorname{meas}_{n} \Omega^{0}+O\left(|\xi|^{1-n}\right) \\
& =-\Phi(\xi) f(0) \operatorname{meas}_{n} \omega_{1}+O\left(|\xi|^{1-n}\right), \quad|\xi| \rightarrow \infty,
\end{aligned}
$$

so that it contributes nontrivially to the boundary condition in (6.7), (6.8). Namely, this condition takes the form

$$
v^{(n)}(x)=-\left(\overline{\mathcal{D}\left(\nabla_{x}\right)} \Phi(x)^{\top}\right)^{\top} P \mathcal{D}\left(\nabla_{x}\right) v_{(0)}(0)+\Phi(x) f(0) \text { meas }_{n} \Omega^{0}, \quad x \in \partial \Xi .
$$

Thus, in formula (6.11), in addition to the previous expression involving the polarization matrix, the expression $\operatorname{Re}\left(\overline{f(0)}^{\top} v_{(0)}(0)\right)$ meas $_{n} \Omega^{0}$ arises. After multiplication by $-\frac{1}{2} h^{n}$ (cf. (6.10) ), this term cancels with the additional summand in the representation of the leading asymptotic term of the energy functional

$$
\begin{aligned}
-\frac{1}{2} \operatorname{Re}\left(f, v_{(0)}\right)_{\Xi(h)} & =-\frac{1}{2} \operatorname{Re}\left(f, v_{(0)}\right)_{\Xi}+\frac{1}{2} \operatorname{Re}\left(f, v_{(0)}\right)_{\omega_{h}} \\
& =E\left(v_{(0)}, f ; \Xi\right)+\frac{1}{2} h^{n} \operatorname{Re}\left(\bar{v}_{(0)}^{\top} f(0)\right) \text { meas }_{n} \Omega^{0}+O\left(h^{n+\alpha}\right) .
\end{aligned}
$$

As a result, we see that the asymptotic formula (6.2) remains valid without changes also for the mixed boundary-value problem (6.12). 
To pose the inclusion optimization problem ("inclusion" should be exchanged for "patch" in the 2-dimensional case), we need to assume some isotropy property of the matrix differential operator $\mathcal{D}\left(\nabla_{x}\right)$. Namely, for any orthogonal transformation

$$
x \mapsto y=\vartheta x
$$

of the Cartesian coordinates with an orthogonal (real unitary) matrix $\vartheta=\left(\vartheta_{p q}\right)$ of size $n \times n$, there should exist unitary matrices $\Theta$ and $\theta$ of size $N \times N$ and $k \times k$, respectively, such that

$$
\mathcal{D}\left(\nabla_{x}\right)=\Theta \mathcal{D}\left(\nabla_{y}\right) \theta
$$

and the mappings

$$
\mathbb{R}^{n \times n} \ni \vartheta \mapsto \Theta \in \mathbb{C}^{N \times N}, \quad \mathbb{R}^{n \times n} \ni \vartheta \mapsto \theta \in \mathbb{C}^{k \times k}
$$

are smooth.

Remark 6.3. 1) Since

$$
\frac{\partial}{\partial x_{j}}=\vartheta_{1 j} \frac{\partial}{\partial y_{1}}+\cdots+\vartheta_{n j} \frac{\partial}{\partial y_{n}}
$$

we have $\nabla_{x}=\vartheta^{\top} \nabla_{y}$; hence, in the case of the scalar operator $\mathcal{L}^{i}\left(\nabla_{x}\right)$ (see Example 1.1), identity (6.14) is fulfilled for $\Theta=\vartheta^{\top}$ and $\theta=1$.

2) In the plane elasticity theory problem (see Example 1.3), identity (6.14) with the matrices

$$
\begin{aligned}
\vartheta & =\theta=\left(\begin{array}{cc}
\cos \phi & \sin \phi \\
-\sin \phi & \cos \phi
\end{array}\right), \quad \phi \in[0,2 \pi), \\
\Theta & =\left(\begin{array}{ccc}
\cos ^{2} \phi & \sin ^{2} \phi & -2^{1 / 2} \cos \phi \sin \phi \\
\sin ^{2} \phi & \cos ^{2} \phi & 2^{1 / 2} \cos \phi \sin \phi \\
2^{1 / 2} \cos \phi \sin \phi & -2^{1 / 2} \cos \phi \sin \phi & \cos ^{2} \phi-\sin ^{2} \phi
\end{array}\right)
\end{aligned}
$$

is checked by direct calculations (see, e.g., [5, §2.1, p. 63]). In the 3-dimensional problem (see Example 1.2) the matrices enlarge and become bulky; they can be found, e.g., in the paper [42].

Thus, the optimization problem mentioned above can be formulated as follows: in a medium $\mathbb{R}^{n}$ with matrix $\mathcal{A}^{0}$, we need to cut a domain $\omega_{h}$ (inclusion, or patch in the 2dimensional case), with fixed shape but arbitrary position, so that, after filling the cavity in $\Xi(h)$ with this inclusion, the energy functional should take its maximal (or minimal) value. Since the medium is homogeneous (the matrix $\mathcal{A}^{0}$ is constant), we should vary only the position of $\omega_{h}$ relative to the axes of the Cartesian coordinate system $x$; this position is described by transformations (6.13) of coordinates and transformations (6.14) of the coefficient matrix of the differential operator $\mathcal{L}^{0}\left(\nabla_{x}\right)$. In other words, it is required to choose the orthogonal matrix $\vartheta$ in formula (6.13) so that the solution $v_{(h)}^{\vartheta} \in \stackrel{\circ}{H}^{0}(\Xi)^{k}$ of problem (2.1) - (2.3), where the coefficient $\mathcal{A}^{1}$ is fixed, and $\mathcal{A}^{0}$ is replaced with $\bar{\vartheta}^{\top} \mathcal{A}^{0} \vartheta$, provide the maximum (minimum) for the functional (2.8).

The dependence of the matrix $\vartheta$, and hence, of the solution $\Theta$, on $\vartheta$ is assumed to be smooth; therefore, the functional has points of global maximum and minimum. However, in the general situation, it is fairly difficult to find them, because the functional in question depends on the solution of the singularly perturbed problem as a whole. If we adopt the asymptotic approach to optimization, i.e., we only study the asymptotic correction term $O\left(h^{n}\right)$ in the expansion (6.2), then the problem simplifies significantly, but still requires solving the family of equations (1.2) with the transmission conditions (1.3), (4.9) for determining the polarization matrix $P^{\vartheta}$. 
For an ellipsoid-shaped inclusion $\omega_{h}$, formula (5.24) makes the inclusion optimization problem entirely algebraic, provided that the matrix $\Psi$ defined by (5.9) is known: it suffices to calculate the stationary points of the sesquilinear form

$$
\begin{aligned}
\vartheta \mapsto & \overline{\mathcal{D}\left(\nabla_{x}\right) v_{(0)}(0)}{ }^{\top}\left(\mathcal{A}^{1}-\bar{\Theta}^{\top} \mathcal{A}^{0} \Theta+\left(\mathcal{A}^{1}-\bar{\Theta}^{\top} \mathcal{A}^{0} \Theta\right) \Psi^{1 / 2}\right. \\
& \left.\times\left(\mathbb{I}_{N}-\Psi^{1 / 2}\left(\mathcal{A}^{1}-\bar{\Theta}^{\top} \mathcal{A}^{0} \Theta\right) \Psi^{1 / 2}\right)^{-1} \Psi^{1 / 2}\left(\mathcal{A}^{1}-\bar{\Theta}^{\top} \mathcal{A}^{0} \Theta\right)\right) \mathcal{D}\left(\nabla_{x}\right) v_{(0)}(0) .
\end{aligned}
$$

Consider the simplest situation: $\omega_{h}=\mathbb{B}_{h}^{n}$ is a ball, $\mathcal{L}^{i}\left(\nabla_{x}\right)$ is a scalar differential operator (see Example 1.1), and $\mathcal{A}^{1}=\mathbb{I}_{n}$, i.e., $\mathcal{L}^{1}=-\nabla_{x}^{\top} \nabla_{x}$ is the Laplacian. All functions are assumed to be real. Since

$$
\Phi(x)= \begin{cases}\left((n-2) \operatorname{meas}_{n-1} \mathbb{S}^{n-1}\right)^{-1}|x|^{2-n} \Phi^{0}(\varphi) & \text { for } n>2, \\ -(2 \pi)^{-1} \ln |x| & \text { for } n=2,\end{cases}
$$

formula (5.9) with $\mathcal{D}\left(\nabla_{x}\right)=\nabla_{x}$ shows that

$$
\Psi=-\int_{\partial \mathbb{B}_{h}^{n}} \nu^{h}(x)\left(\nabla_{x} \Phi(x)\right)^{\top} d s_{x}=\left(\operatorname{meas}_{n-1} \mathbb{S}^{n-1}\right)^{-1} \int_{\partial \mathbb{S}_{h}^{n-1}} \frac{x}{h}\left(\frac{x}{|x|^{n-2}}\right)^{\top} d s_{x}=\mathbb{I}_{N} .
$$

Then, since $\vartheta^{-1}=\vartheta^{\top}$ for orthogonal matrices, we see that the quadratic form (6.15) can be rewritten as follows:

$$
\begin{aligned}
\nabla_{x} v_{(0)} & (0)^{\top}\left(\mathbb{I}_{n}-\vartheta^{\top} \mathcal{A}^{0} \vartheta+\left(\mathbb{I}_{n}-\vartheta^{\top} \mathcal{A}^{0} \vartheta\right)\left(\mathbb{I}_{n}-\mathbb{I}_{n}+\vartheta^{\top} \mathcal{A}^{0} \vartheta\right)\right)^{-1} \\
& \left.\times\left(\mathbb{I}_{n}-\vartheta^{\top} \mathcal{A}^{0} \vartheta\right)\right) \nabla_{x} v_{(0)}(0) \\
= & \left(\vartheta \nabla_{x} v_{(0)}(0)\right)^{\top}\left(\left(\mathcal{A}^{0}\right)^{-1}-\mathbb{I}_{n}\right) \vartheta \nabla_{x} v_{(0)}(0) .
\end{aligned}
$$

Consequently,

$$
\begin{aligned}
E\left(v_{(h)}, f ; \Xi(h) \cup \omega_{h}\right) \\
=\frac{1}{2}\left(\int_{\Xi(h)}\left|\nabla_{x} v_{(h)}(x)\right|^{2} d x+\int_{\omega_{h}}\left(\vartheta \nabla_{x} v_{(h)}(x)\right)^{\top} \mathcal{A}^{0} \vartheta \nabla_{x} v_{(h)}(x) d x\right)-\int_{\Xi} v_{(h)}(x) f(x) d x \\
=\frac{1}{2} \int_{\Xi}\left|\nabla_{x} v_{(0)}(x)\right|^{2} d x-\int_{\Xi} v_{(0)}(x) f(x) d x \\
\quad+\frac{1}{2} h^{n} \operatorname{meas}_{n} \omega_{1}\left(\left(\vartheta \nabla_{x} v_{(0)}(0)\right)^{\top}\left(\mathcal{A}^{0}\right)^{-1} \vartheta \nabla_{x} v_{(0)}(0)-\left|\nabla_{x} v_{(0)}(0)\right|^{2}\right)+\widetilde{E}(h) .
\end{aligned}
$$

We summarize. In order to maximize (minimize) the main asymptotic correction term $O\left(h^{n}\right)$, the orthogonal coordinate change in the functional (6.2) must be chosen so that the column $\vartheta \nabla_{x} v_{(0)}(0)$ be an eigenvector for the matrix $\mathcal{A}^{0}$ corresponding to its smallest (largest) eigenvalue. Of course, this can be done in the case where $\nabla_{x} v_{(0)}(0) \neq 0 \in \mathbb{R}^{n}$.

Theorem 6.1. Let $\omega_{h}=\mathbb{B}_{h}^{n}$ be a ball, and let $\mathcal{D}\left(\nabla_{x}\right)=\nabla_{x}$. Suppose that, in the scalar differential operators (1.1), the matrices $\mathcal{A}^{1}=\mathbb{I}_{n}$ and $\vartheta^{\top} \mathcal{A}^{0} \vartheta$ are given. Then, for all orthogonal $(n \times n)$-matrices $\vartheta$, the maximal (minimal) value of the functional (2.8) evaluated at the solution $v_{(h)} \in \stackrel{\circ}{H}^{1}(\Xi)$ of problem (2.1)-(2.3) with right-hand side $f \in C^{0, \alpha}(\Xi)$ is equal to

$$
E\left(v_{(h)}, f ; \Xi(h) \cup \omega_{h}\right)=E\left(v_{(0)}, f ; \Xi\right)+\frac{1}{2} h^{n}\left(\frac{1}{\Lambda}-1\right)\left|\nabla_{x} v_{(0)}(0)\right|^{2}+O\left(h^{n+\alpha}\right),
$$

where $v_{(0)} \in C^{2, \alpha}(\Xi)$ is the solution of the limiting problem (6.1), and $\Lambda>0$ is the smallest (largest) eigenvalue of the matrix $\mathcal{A}^{0}$.

In the plane elasticity problem (see Example1.3), the fundamental matrix (the KelvinSomiliana tensor) is known for an arbitrary homogeneous anisotropic medium (see [43]), but simple calculations and the compact answer (6.16) are impossible even in the isotropic 
case, requiring the invocation of a computer. In 3-dimensional elasticity theory (see Example 1.2), the fundamental matrix is found explicitly only for the transversallyisotropic media [44, 45] and for media algebraically equivalent to them [42]. Also, we cannot expect any explicit formula such as (6.17). Nevertheless, in the situation listed above, the optimization problem for inclusions or patches becomes purely algebraic.

\section{REFERENCES}

[1] J. Nečas, Les méthodes directes en théorie des équations elliptiques, Masson et Cie, Paris; Academia, Prague, 1967. MR0227584 (37:3168)

[2] S. A. Nazarov, Self-adjoint elliptic boundary-value problems. The polynomial property and formally positive operators, Probl. Mat. Anal., No. 16, S.-Peterburg. Gos. Univ., St. Petersburg, 1997, pp. 167-192; English transl., J. Math. Sci. (New York) 92 (1998), no. 6, 4338-4353. MR1668418 (2000a:35042)

[3] , Polynomial property of selfadjoint elliptic boundary value problems and the algebraic description of their attributes, Uspekhi Mat. Nauk 54 (1999), no. 5, 77-142; English transl., Russian Math. Surveys 54 (1999), no. 5, 947-1014. MR1741662 (2001k:35073)

[4] S. G. Lekhnitskiǔ, Theory of elasticity of an anisotropic body, 2nd ed., Nauka, Moscow, 1977; English transl. of 1st ed., Holden-Day, Inc., San Francisco, 1963. MR0502604(58:575) MR0180018 $(31: 4255)$

[5] S. A. Nazarov, Asymptotic theory of thin plates and rods. Dimension reduction and integral estimates, Nauchn. Kniga, Novosibirsk, 2002. (Russian)

[6] G. Pólya and G. Szegö, Isoperimetric inequalities in mathematical physics, Ann. of Math. Stud., No. 27, Princeton Univ. Press, Princeton, NJ, 1951. MR0043486 (13:270d)

[7] I. S. Zorin, A. B. Movchan, and S. A. Nazarov, Application of tensors of elastic capacity, polarization and associated deformation, Issled. po Uprugosti i Plastichnosti, No. 16, Leningrad. Univ., Leningrad, 1990, pp. 75-91. (Russian) MR.1070591(91k:73037)

[8] S. A. Nazarov, The damage tensor and measures. I. Asymptotic analysis of anisotropic media with defects, Izv. Ross. Akad. Nauk Mekh. Tverd. Tela 2000, no. 3, 113-124; English transl., Mech. Solids 35 (2000), no. 3, 96-105.

[9] _ Asymptotic conditions at a point, selfadjoint extensions of operators, and the method of matched asymptotic expansions, Trudy S.-Peterburg. Mat. Obshch. 5 (1998), 112-183; English transl., Amer. Math. Soc. Transl. Ser. 2, vol. 193, Amer. math. Soc., Providence, RI, 1999, pp. 77125. MR:1736907 (2000m:35016)

[10] A. B. Movchan, Polarization of Wiener capacity matrices of an operator in elasticity theory in doubly connected domains, Mat. Zametki 47 (1990), no. 2, 151-153; English transl. in Math. Notes 47 (1990), no. 2. MR 1048555 (91i:73009)

[11] _ Integral characteristics of elastic inclusions and cavities in the two-dimensional theory of elasticity, European J. Appl. Math. 3 (1992), 21-30. MR1156592 (93c:73023)

[12] A. B. Movchan and N. V. Movchan, Mathematical modelling of solids with nonregular boundaries, CRC Press, Boca Raton, FL, 1995. MR.1384979 (97e:73001)

[13] I. I. Argatov, Integral characteristics of rigid inclusions and cavities in the two-dimensional theory of elasticity, Prikl. Mat. Mekh. 62 (1998), no. 2, 283-289; English transl., J. Appl. Math. Mech. 62 (1998), no. 2, 266-268. MR1680676 (99m:73010)

[14] J. D. Eshelby, The determination of the elastic field of an ellipsoidal inclusion, and related problems, Proc. Roy. Soc. London Ser. A 241 (1957), 376-396. MR.0087326 (19:338d)

[15] I. A. Kunin, Elastic media with microstructure. II. Three dimensional models, Springer Ser. SolidState Sci., vol. 44, Springer-Verlag, Berlin, 1983. MR0710127 (85d:73030)

[16] S. K. Kanaun and V. M. Levin, The effective field method in the mechanics of composite materials, Petrozavod. Univ., Petrozavodsk, 1993. (Russian) MR1330223(96a:73050)

[17] I. A. Kunin and È. G. Sosnina, Ellipsoidal inhomogeneity in elastic medium, Dokl. Akad. Nauk SSSR 199 (1971), no. 3, 571-574. (Russian)

[18] _ Stress concentration on ellipsoidal inhomogeneity in an anisotropic elastic medium, Prikl. Mat. Mekh. 37 (1973), no. 2, 306-315. (Russian)

[19] T. Mura, Micromechanics of defects in solids, 2nd ed., Kluwer, Dordrecht, 1987.

[20] A. B. Freidin, On new phase inclusions in elastic solids, ZAMM 87 (2007), no. 2, 102-116. MR 2298654 (2008c:74003)

[21] A. B. Freidin and E. N. Vilchevskaya, Multiple development of new phase inclusions in elastic solids, Internat. J. Engrg. Sci. 47 (2009), no. 2, 240-260. MR2498974 (2010e:74071) 
[22] O. A. Ladyzhenskaya, Boundary value problems of mathematical physics, Nauka, Moscow, 1973; English transl., Appl. Math. Sci., vol. 49, Springer, New York, 1985. MR0599579 (58:29032) MR0793735 (87f:35001)

[23] J.-L. Lions and E. Magenes, Problèmes aux limites non homogènes et applications. Vol. 1, Travaux et Recherches Math., No. 17, Dunod, Paris, 1968. MR0247243 (40:512)

[24] V. A. Kondrat'ev, Boundary value problems for elliptic equations in domains with conical or angular points, Trudy Moskov. Mat. Obshch. 16 (1967), 209-292; English transl. in Trans. Moscow Math. Soc. 1967 (1968). MR 0226187 (37:1777)

[25] S. A. Nazarov and B. A. Plamenevskiŭ, Elliptic problems in domains with piecewise smooth boundaries, Nauka, Moscow, 1991; English transl., de Gruyter Exp. Math., vol. 13, Walter de Gruyter and Co., Berlin, 1994. MR 1283387 (95h:35001)

[26] A. Pazy, Asymptotic expansions of solutions of ordinary differential equations in Hilbert space, Arch. Rational Mech. Anal. 24 (1967), 193-218. MR.0209618(35:515)

[27] I. M. Gel'fand and G. E. Shilov, Generalized functions and operations on them, Fizmatgiz, Moscow, 1958; English transl., Generalized functions. Vol. 1. Properties and operations, Acad. Press, New York-London, 1964 [1977]. MR0097715(20:4182) MR0435831 (55:8786a)

[28] V. G. Maz'ya and B. A. Plamenevskiř, The coefficients in the asymptotics of solutions of elliptic boundary value problems with conical points, Math. Nachr. 76 (1977), 29-60. (Russian) MR0601608 $(58: 29176)$

[29] Estimates in $L_{p}$ and in Hölder classes, and the Miranda-Agmon maximum principle for the solutions of elliptic boundary value problems in domains with singular points on the boundary, Math. Nachr. 81 (1978), 25-82. (Russian) MR0492821 (58:11886)

[30] M. Lobo, S. A. Nazarov, and E. Perez, Eigen-oscillations of contrasting non-homogeneous elastic bodies: Asymptotic and uniform estimates for eigenvalues, IMA J. Appl. Math. 70 (2005), no. 3, 419-458. MR.2144635 (2006g:74024)

[31] Ya. A. Roŭtberg, Elliptic boundary value problems in the spaces of distributions, Math. Appl., vol. 384, Kluwer Acad. Publ. Group, Dordrecht, 1996. MR.1423135 (97m:35055)

[32] S. G. Mikhlin, Higher-dimensional singular integrals and integral equations, Fizmatgiz, Moscow, 1962. (Russian) MR0155165 (27:5105)

[33] S. Agmon, A. Douglis, and L. Nirenberg, Estimates near the boundary for solutions of elliptic partial differential equations satisfying general boundary conditions. II, Comm. Pure Appl. Math. 17 (1964), no. 1, 35-92. MR0162050 (28:5252)

[34] Ya. A. Roĭtberg and Z. G. Sheftel', General boundary-value problems for elliptic equations with discontinuous coefficients, Dokl. Akad. Nauk SSSR 148 (1963), no. 5, 1034-1037; English transl. in Soviet Math. Dokl. 4 (1963). MR0146507 (26:4029)

[35] A. M. Il'in, Matching of asymptotic expansions of solutions of boundary value problems, Nauka, Moscow, 1989; English transl., Transl. Math. Monogr., vol. 102, Amer. Math. Soc., Providence, RI, 1992. MR1007834 (90i:35062), MR1182791 (93g:35016)

[36] W. G. Maz'ya, S. A. Nasarow, and B. A. Plamenewski, Asymptotische Theorie elliptischer Randwertaufgaben in singulär gestörten Gebieten. I, Math. Lehrbücher Monogr. II. Abt. Math. Monogr., Bd. 82, Akademie-Verlag, Berlin, 1991; English transl., Maz'ya V., Nazarov S., and Plamenevskiü B., Asymptotic theory of elliptic boundary value problems in singularly perturbed domains. Vol. 1, Oper. Theory Adv. Appl., vol. 111, Birkhäuser-Verlag, Basel, 2000. MR1101139 (92g:35059) MR.1779977 (2001e:35044a)

[37] V. G. Maz'ya and S. A. Nazarov, Asymptotic behavior of energy integrals under small perturbations of the boundary near corner and conic points, Trudy Moskov. Mat. Obshch. 50 (1987), 79-129; English transl., Trans. Moscow Math. Soc. 1988, 77-127. MR0912054 (89a:35034)

[38] S. A. Nazarov and M. Specovius-Neugebauer, Approximation of unbounded domains by bounded domains. Boundary value problems for the Lamé operator, Algebra i Analiz 8 (1996), no. 5, 229268; English transl., St. Petersburg Math. J. 8 (1997), no. 5, 879-912. MR.1428992 (97m:65234)

[39] S. A. Nazarov and J. Sokolowski, Asymptotic analysis of shape functionals, J. Math. Pures Appl. (9) 82 (2003), no. 2, 125-196. MR1976204 (2004c:35015)

[40] S. A. Nazarov and M. Specovius-Neugebauer, Approximation of exterior problems. Optimal conditions for the Laplacian, Analysis 16 (1996), no. 4, 305-324. MR.1429456 (97j:35017)

[41] S. A. Nazarov and J. Sokolowski, Self-adjoint extensions for the Neumann Laplacian and applications, Acta Math. Sin. (Engl. Ser.) 22 (2006), no. 3, 879-906. MR2220182 (2007a:49073)

[42] S. Langer, S. A. Nazarov, and M. Specovius-Neugebauer, Affine transformations of threedimensional anisotropic media, and explicit formulas for fundamental matrices, Prikl. Mekh. Tekhn. Fiz. 47 (2006), no. 2, 95-102; English transl., J. Appl. Mech. Tech. Phys. 47 (2006), no. 2, 229-235. MR2210443(2007a:74039) 
[43] V. D. Kupradze, T. G. Gegeliya, M. O. Basheleǔshvili, and T. V. Burchuladze, Three-dimensional problems of the mathematical theory of elasticity and thermoelasticity, Nauka, Moscow, 1976; English transl., North-Holland Ser. in Appl. Math. Mech., vol. 25, North-Holland Publ. Co., Amsterdam-New York, 1979. MR0421228 (54:9233) MR0530377 (80h:73002)

[44] I. M. Lifshits and L. N. Rozentsverg, On the construction of the Green's tensor for the fundamental equations of the theory of elasticity in the case of an unrestricted elastic-anisotropic medium, Zh. Èksper. Teoret. Fiz. 17 (1947), 783-791. (Russian) MR0022520 (9:219b)

[45] E. Kröner, Das Fundamentalintegral der anisotropen elastischen Differentialgleichungen, Z. Physik 136 (1953), 402-410. MR0064606 (16:308e)

Institute of Mechanical Engineering Problems, Bol'shoi Prospekt V.O. 61, St.Petersburg 199178, Russia

E-mail address: srgnazarov@yahoo.co.uk

Received 24/MAR/2009

Translated by A. PLOTKIN 